\title{
The ROSAT-ESO flux limited X-ray (REFLEX) galaxy cluster survey. I. The construction of the cluster sample ${ }^{\star}$
}

\author{
1 Max-Planck-Institut für extraterr. Physik, D 85740 Garching, Germany \\ 2 Osservatorio Astronomico di Brera, Merate, Italy \\ 3 Liverpool John Moores University, Liverpool,UK \\ ${ }^{4}$ CEA Saclay, Service d'Astrophysique, Gif-sur-Yvette, France \\ 5 Naval Research Laboratory, Washington, USA \\ 6 Dipartimento di Fisica, Universita degli Studi di Milano, Italy \\ 7 Physics Department, University of Durham, UK \\ 8 Royal Observatory, Edinburgh, UK \\ 9 European Southern Observatory, Garching, Germany
}

H. Böhringer ${ }^{1}$, P. Schuecker ${ }^{1}$, L. Guzzo ${ }^{2}$, C. A. Collins ${ }^{3}$, W. Voges ${ }^{1}$, S. Schindler ${ }^{3}$, D. M. Neumann ${ }^{4}$, R. G. Cruddace ${ }^{5}$, S. De Grandi ${ }^{2}$, G. Chincarini ${ }^{2,6}$, A. C. Edge $^{7}$, H. T. MacGillivray ${ }^{8}$, and P. Shaver ${ }^{9}$

Received 29 August 2000 / Accepted 7 February 2001

\begin{abstract}
We discuss the construction of an X-ray flux-limited sample of galaxy clusters, the REFLEX survey catalogue, to be used for cosmological studies. This cluster identification and redshift survey was conducted in the frame of an ESO key programme and is based on candidates selected from the southern part of the ROSAT All-Sky Survey (RASS). For the first cluster candidate selection from a flux-limited RASS source list, we make use of optical data from the COSMOS digital catalogue produced from the scans of the UK-Schmidt plates. To ensure homogeneity of the sample construction process, this selection is based only on this one well-defined optical data base. The nature of the candidates selected in this process is subsequently checked by a more detailed evaluation of the X-ray and optical source properties and available literature data. The final identification and the redshift is then based on optical spectroscopic follow-up observations.

In this paper we document the process by which the primary cluster candidate catalogue is constructed prior to the optical follow-up observations. We describe the reanalysis of the RASS source catalogue which enables us to impose a proper flux limit cut to the X-ray source list without introducing a severe bias against extended sources. We discuss the correlation of the X-ray and optical (COSMOS) data to find galaxy density enhancements at the RASS X-ray source positions and the further evaluation of the nature of these cluster candidates. Based also on the results of the follow-up observations we provide a statistical analysis of the completeness and contamination of the final cluster sample and show results on the cluster number counts. The final sample of identified X-ray clusters reaches a flux limit of $310^{-12} \mathrm{erg} \mathrm{s}^{-1} \mathrm{~cm}^{-2}$ in the $0.1-2.4 \mathrm{keV}$ band and comprises 452 clusters in an area of 4.24 ster. The results imply a completeness of the REFLEX cluster sample well in excess of $90 \%$. We also derive for the first time an upper limit of less than $9 \%$ for the number of clusters which may feature a dominant contribution to the X-ray emission from AGN. This accuracy is sufficient for the use of this cluster sample for cosmological tests.
\end{abstract}

Key words. cosmology: miscellaneous - galaxies: clusters - X-rays: galaxies

\section{Introduction}

Clusters of galaxies as the largest well-defined building blocks of our Universe are ideal probes for the study of the cosmic large-scale structure. Statistical measures of the galaxy cluster population like the cluster mass func-

\footnotetext{
Send offprint requests to: H. Böhringer,

e-mail: hxb@mpe.mpg.de

* Based on observations at the European Southern Observatory La Silla, Chile.
}

tion (e.g. Press \& Schechter 1974; Kaiser 1986; Henry \& Arnaud 1991; Böhringer \& Wiedenmann 1992; White et al. 1993; Bahcall \& Cen 1992; Oukbir \& Blanchard 1992, 1997; Eke et al. 1996; Viana \& Liddle 1996; Borgani et al. 1999), functions describing the spatial distribution as the two-point-correlation function (e.g. Bahcall \& Soneira 1983; Klypin \& Kopilev 1983; Bahcall 1988; Lahav et al. 1989; Nichol et al. 1992; Dalton et al. 1994; Romer et al. 1994; Abadi et al. 1998; Borgani et al. 1999; Moscardini et al. 2000; Collins et al. 2000, Paper II in this series) 
and the density fluctuation power spectrum (e.g. Peacock \& West 1992; Einasto et al. 1997; Retzlaff et al. 1998; Tadros et al. 1998; Miller \& Batuski 2000; Schuecker et al. 2000, Paper III in this series), can place very important constraints on the characteristic measures of the matter density distribution throughout the Universe and its evolution as a function of time. This is due to the fact that the formation of galaxy clusters is tightly linked to the formation of the large scale structure in our Universe as a whole. That clusters are indeed good tracers of the largescale structure is discussed and demonstrated further in a following papers by Schuecker et al. (2000).

The crucial step in any of these studies is the careful primary selection of the galaxy cluster sample to be used for the cosmological investigation. Ideally one would like to select the clusters by their mass, thus defining the sample by all clusters above a certain mass limit. This parameter is also the most direct parameters predicted by analytical cosmological models (e.g. Press-Schechter 1974 type models) or by $N$-body simulations (e.g. Frenk et al. 1990; Cen \& Ostriker 1994; Kofman et al. 1996; Bryan \& Norman 1998; Thomas et al. 1998; Frenk et al. 1999). The cluster mass is not easily and directly obtained from observations, however. Thus one has to resort to observable criteria, which should be as closely linked to the mass of the clusters as possible.

Since galaxy clusters were first discovered by their galaxy density enhancements, a galaxy richness criterion was first used to define and select clusters of galaxies. The first large and very widely used compilation was that of Abell (1958) and Corwin \& Olowin (Abell et al. 1989) whose selection criteria were fixed to a minimal galaxy number density within a metric radius of $3 h_{50}^{-1} \mathrm{Mpc}$ and a defined magnitude interval. This catalogue was compiled by eye inspection of the Palomar Sky Survey Plates and subsequently of UK Schmidt survey plates. Another comprehensive cluster catalogue was compiled visually by Zwicky and collaborators (Zwicky et al. 196168 ) with a significantly different cluster definition. Later, similar cluster catalogues were constructed based on machine work using digitized data from scans of the optical plates (by COSMOS, see Heydon-Dumbleton et al. 1989; Lumbsden et al. 1992 and APM; see Maddox et al. 1990; Dalton et al. 1997) using more objective criteria. Further improvement in the optical cluster searches was achieved by using multicolor CCD surveys and matched filter techniques (e.g. Postman et al. 1996; Olsen et al. 1999). But it is still very difficult and uncertain to assign a mass to a cluster with a given observed richness without comprehensive redshift data.

One of the main problems in assigning a richness to a galaxy cluster in the optical is the fact that the cluster is seen against a background galaxy distribution which is far from being homogeneous but shows structure on all scales. The latter effect is clearly shown by the autocorrelation analysis of the two-dimensional projected galaxy distribution on the sky. It is therefore difficult to determine a background-subtracted galaxy number of a cluster in a unique fashion. Also, the so-called projection effects, in which several galaxy groups or a filamentary structure in the line of sight can mimic a compact rich cluster, are basically a result of this inhomogeneous matter distribution (e.g. van Haarlem 1997).

The possibility of detecting galaxy clusters in X-rays has since been recognized as a way to improve the unambiguity of the detection. The X-ray emission observed in clusters originates from the thermal emission of hot intracluster gas (e.g. Sarazin 1986) which is distributed smoothly throughout the cluster. The plasma is bound by the gravitational potential well of the clusters and fills the potential approximately in a hydrostatic fashion. Therefore the plasma emission is a very good tracer of the cluster's gravitational potential. Even though the plasma is very tenuous, the large volume makes galaxy clusters the most luminous X-ray sources besides AGN. In addition the thermal emission for the typical intracluster plasma temperatures of several $\mathrm{keV}$ has the spectral maximum in the soft X-ray band where the available X-ray telescopes are most effective. This makes galaxy clusters readily detectable out to large distances with present X-ray telescopes.

However, the main advantage of the X-ray detection is the fact that the X-ray luminosity is closely correlated to the cluster mass (Reiprich \& Böhringer 1999), with a dispersion of about $50 \%$ in the determination of the mass for a given X-ray luminosity (Reiprich \& Böhringer, in preparation). Thus, in summary X-ray selection provides the following positive features:

- An effective selection by mass (with a known dispersion which can be taken into account in any corresponding modeling).

- The X-ray background originates mostly from distant point sources which are very homogeneously distributed (e.g. Soltan \& Hasinger 1994). Therefore the X-ray background is very much easier to subtract from the cluster emission than the optical galaxy background distribution. - The X-ray surface brightness is much more concentrated towards the cluster centre as compared to the galaxy distribution. Therefore the effect of overlaps along the line of sight is minimized.

- For an X-ray flux-limited survey the survey volume as a function of X-ray luminosity can exactly be calculated (e.g. for the construction of the X-ray luminosity or mass function).

The construction of statistically complete samples of $\mathrm{X}$-ray clusters started with the completion of the first allsky X-ray surveys by the HEAO-1 and ARIEL V satellites (Piccinotti et al. 1982; Kowalski et al. 1984). With additional observations from EINSTEIN and EXOSAT a cluster sample of the $\sim 50$ X-ray brightest objects with more detailed X-ray data was compiled (Lahav et al. 1989; Edge et al. 1990) and with the analysis of deeper EINSTEIN observations the first deep X-ray cluster survey, within the EMSS, has been obtained (Gioia et al. 1990; Henry et al. 1992). The latter survey allowed in particular to address the question of the evolution of cluster 
abundance with redshift (e.g. Henry et al. 1992; Nichol et al. 1997). The ROSAT All-Sky Survey (RASS), the first X-ray all-sky survey conducted with an X-ray telescope (Trümper 1992, 1993) provides an ideal basis for the construction of a large X-ray cluster sample for cosmological studies. Previous cluster surveys based on the RASS include: Romer et al. (1994); Pierre et al. (1994); Burns et al. (1996); Ebeling et al. (1996, 1998, 2000a, 2000b); De Grandi (1999); Henry et al. (1997); Ledlow et al. (1999); Böhringer et al. (2000); and Cruddace et al. (2000). Two of these surveys are pilot projects to REFLEX concentrating on the South Galactic Pole and the Hydra regions with results reported in Romer et al. (1994, see also Cruddace 2001) and Pierre et al. (1994), respectively. The sample described by De Grandi et al. (1999) was compiled from an earlier version of the current cluster sample based on X-ray data from the first processing of the RASS and a significantly shallower correlation with the COSMOS data base as well as correlations with a variety of optical cluster catalogues. It constitutes a subsample of the present cluster sample comprising 130 clusters at a flux limit of $3-410^{-12} \mathrm{erg} \mathrm{s}^{-1} \mathrm{~cm}^{-2}$ (as measured in the $0.5-2 \mathrm{keV}$ energy band) in $2.5 \mathrm{sr}$ of the southern sky. The work reported in Cruddace et al. (2001) uses the same starting material as the present work with a slightly different cluster search method applied to the COSMOS data and goes deeper in flux in an area limited to 1.013 ster around the South Galactic Pole. The cluster samples described in Ebeling et al. (1996); Burns et al. (1996), and Ledlow et al. (1999) are derived from correlations of the cluster catalogue by Abell et al. (1989) with the RASS data. The work described in Henry et al. (1997) concentrates on a small area around the North Ecliptic Pole with the special feature of this survey that all Xray sources, not only the clusters, are identified up the flux-limit of the sample. The northern BCS survey (Allen et al. 1992; Crawford et al. 1995, 1999; Ebeling et al. 1998, 2000a) is optimising the search for clusters by combining the correlation with several optical catalogues, by relying on X-ray extent information, and by combining two different detection algorithms - the standard RASS processing for the complete region and the Voronoi Tesselation and Percolation method covering about one seventh of the survey area. The price payed for the application of several, partly inhomogeneous selection processes in parallel is an inhomogeneous selection function which is very hard to specify and no details have been published up to date.

The most important final goal of the present survey is the statistical and cosmographical characterisation of the large-scale structure of the present day Universe. This requires a large enough sample by number and volume and a nearly homogeneous and well controlled selection function in order to minimize and correct for any artificial fluctuations in the cluster density. The first condition is not provided by the above surveys concentrating on a small sky area while the latter point is not fulfiled by the surveys based on optical catalogues (e.g. the Abell catalogue) with known selection problems and inhomogeneous source detection as featured by the early RASS processing or reanalysis covering only part of the sky. Therefore, with the current survey (and its complement in the South Galactic Pole region by Cruddace et al.), we are following a completely new avenue using a highly homogeneous sampling of information from the X-ray RASS II data and the COSMOS optical data base. The importance and success of this new approach is demonstrated, for example, by preempting the results derived in this paper and comparing the sky surface density of the present cluster sample with the northern BCS sample: at the flux-limit of BCS, the BCS sample reaches $78 \%$ of the surface density of clusters in the present sample (see Sect. 11 for details). This reduction in incompleteness is expected to go along with an increase in homogeneity.

For the construction of the present cluster sample optical, follow-up observations, in addition to the X-ray analysis and X-ray/optical correlations, are necessary to clearly identify the nature of the X-ray sources and to determine the cluster redshifts. To this aim we have conducted an intensive follow-up optical survey project as an ESO key program from 1992 to 1999 (e.g. Böhringer 1994; Guzzo et al. 1995; Böhringer et al. 1998; Guzzo et al. 1999) which has been termed REFLEX (ROSAT-ESO-Flux-LimitedX-ray) Cluster Survey. Within this program the identification of all the cluster candidates at $\delta \leq 2.5 \mathrm{deg}$ and down to a flux limit of $310^{-12} \mathrm{erg} \mathrm{s}^{-1} \mathrm{~cm}^{-2}$ in the ROSAT band ( 0.1 to $2.4 \mathrm{keV}$ ) has been completed. This sample includes 452 identified galaxy clusters, 449 of which have a measured redshift. An extension of the identification programme down to a lower flux limit has been started and a large number of redshifts for this extension has already been secured.

A complementary RASS cluster redshift survey programme is conducted for the northern celestial hemisphere in a collaboration by MPE, STScI, CfA, and ESO, the Northern ROSAT All-Sky Cluster Survey (NORAS; e.g. Böhringer 1994; Burg et al. 1994) and a first catalogue containing 483 identified X-ray galaxy clusters has recently been published (Böhringer et al. 2000). It is the future aim to combine the northern and southern surveys which in our ongoing program are based on slightly different identification strategies, mostly due to the different optical data available. Work on homogenization of this is in progress. We have also successfully extended the cluster search into the region close to the galactic plane covering about $2 / 3$ of the region with galactic latitude $\left|b_{\text {II }}\right|<20 \mathrm{deg}$ (Böhringer et al. 2001b).

In this paper, we describe the selection of the cluster candidate sample for the REFLEX Survey. The layout of the paper is as follows. In Sect. 2 we characterize the depth and the sky area of the study and in Sect. 3 the basic RASS data used as input. It was found that a reanalysis of the $\mathrm{X}$-ray properties of the clusters in the RASS was necessary for the project. This new reanalysis technique and its results are presented in Sect. 4. The selection of the galaxy cluster candidates by means of a correlation of the X-ray source positions with the optical data base from COSMOS 
Table 1. Regions of the sky at the LMC and SMC excised from the Survey

\begin{tabular}{llllll}
\hline region & RA & range & DEC & range & area (ster) \\
\hline LMC1 & 58 & $\rightarrow 103^{\circ}$ & -63 & $\rightarrow-77^{\circ}$ & 0.0655 \\
LMC2 & 81 & $\rightarrow 89^{\circ}$ & -58 & $\rightarrow-63^{\circ}$ & 0.0060 \\
LMC3 & 103 & $\rightarrow 108^{\circ}$ & -68 & $\rightarrow-74^{\circ}$ & 0.0030 \\
SMC1 & 358.5 & $\rightarrow 20^{\circ}$ & -67.5 & $\rightarrow-77^{\circ}$ & 0.0189 \\
SMC2 & 356.5 & $\rightarrow 358.5^{\circ}$ & -73 & $\rightarrow-77^{\circ}$ & 0.0006 \\
SMC3 & 20 & $\rightarrow 30^{\circ}$ & -67.5 & $\rightarrow-72^{\circ}$ & 0.0047 \\
\hline
\end{tabular}

is described in Sects. 5 and 6. The further X-ray source classification is discussed in Sect. 7. Section 8 provides tests of the sample completeness. The resulting REFLEX cluster sample for a flux limit of $310^{-12} \mathrm{erg} \mathrm{s}^{-1} \mathrm{~cm}^{-2}$ $(0.1-2.4 \mathrm{keV})$ and some of its characteristics is described in Sect. 9. Further statistics of the X-ray properties of the REFLEX clusters and the contamination of the sample by non-cluster sources is discussed in Sect. 10, and Sect. 11 provides a summary and conclusions.

\section{The REFLEX galaxy cluster survey}

The survey area of REFLEX is the southern hemisphere below a declination of $+2.5 \mathrm{deg}$. A region of \pm 20 degrees around the galactic plane is excluded form the study, since clusters are difficult to recognize optically in the dense stellar fields of the Milky Way and the X-ray detection is hampered by the higher interstellar absorption in the inner parts of the galactic band. The region 2.5 degrees above the equatorial equator is included in this project since the COSMOS data extend up to this declination. It provides some overlap with the NORAS survey project (e.g. Böhringer et al. 2000) where both cluster identification programmes can be compared. The total area thus covered is 4.34 ster or $14248 \mathrm{deg}^{2}$.

In addition to the region around the galactic plane, the dense stellar fields of the two Magellanic clouds prevent an efficient galaxy search in these regions of the southern sky. In particular the star-galaxy separation technique used in the construction of the COSMOS data base became inefficient in these crowded areas (H.T. MacGillivray, private communication) and therefore no star-galaxy classification is actually provided in the COSMOS data released and used for our project. Therefore we exclude an area of $244.4 \mathrm{deg}^{2}$ for the LMC and $79.8 \mathrm{deg}^{2}$ for the SMC which essentially follows the boundaries of those UK-Schmidt plates without object classification. The areas which are excised from our survey are specified in detail in Table 1.

The total survey area after this excision amounts to 4.24 ster or $13924 \mathrm{deg}^{2}$ which corresponds to $33.75 \%$ of the sky. This survey covers the largest area for which currently a homogeneous combined optical/X-ray survey is possible, since there is no optical survey covering both hemispheres simultaneously.
The observational goal of this survey programme is the identification and redshift determination of all galaxy clusters in the study area above a given flux limit. In a first step, within the ESO key programme, we have completed the observations for a sample of 452 galaxy cluster (with redshifts for 449 clusters) above a limiting flux of $310^{-12} \mathrm{erg} \mathrm{s}^{-1} \mathrm{~cm}^{-2}(0.1-2.4 \mathrm{keV})$. In addition we have already secured many redshifts at lower fluxes and we plan to extend the redshift survey to flux limit of $1.6-210^{-12} \mathrm{erg} \mathrm{s}^{-1} \mathrm{~cm}^{-2}$. This corresponds to a count rate limit in the hard ROSAT band of about $0.08-0.1 \mathrm{cts} \mathrm{s}^{-1}$. With a typical exposure in the southern part of the RASS of about 330 sec this yields about 2530 photons for the fainter sources. This is still just enough to determine a flux within uncertainty limits of typically less than $30 \%$ and provides some leverage for the determination of some source properties. At this flux limit we expect between 700 and 1000 galaxy clusters in the survey area (based on the number counts of previous surveys e.g. Gioia et al. 1990; Rosati et al. 1998).

For the preparation of the candidate sample we have therefore chosen to start with a source sample with a count rate limit of $0.08 \mathrm{cts} \mathrm{s}^{-1}$ in the hard ROSAT band (channel 52 to 201 corresponding approximately to an energy range of 0.5 to $2.0 \mathrm{keV}$ ). Note that all the fluxes quoted in this paper refer to the total ROSAT energy band $(0.1-2.4 \mathrm{keV})$ in contrast to the more restricted band of pulse high channels chosen for the determination of the count rate. This count rate limit translates into a flux limit for cluster type spectra of 1.55-1.95 $10^{-12} \mathrm{erg} \mathrm{s}^{-1} \mathrm{~cm}^{-2}$, a range determined mainly by variations of the interstellar HI column density in the REFLEX area (20\% in the range $\left.1-1010^{20} \mathrm{~cm}^{-2}\right)$. Weaker dependences on the cluster temperature (e.g. $\sim 1.4 \%$ in the range $3-8 \mathrm{keV}$, see Fig. 8 in Böhringer et al. 2000) and redshift (in analogy to the optical K-correction, $0.5 \%$ in the range $z=0$ to $z=0.2$ ) are found. (Below about $2 \mathrm{keV}$ the temperature dependence is stronger, however.) We will be quoting unabsorbed flux values in the ROSAT energy band (defined as 0.1 to $2.4 \mathrm{keV}$ ) throughout this paper since the results in this energy band are less dependent on the spectral model assumptions for the sources compared to any other significantly wider band definition. Further assumptions or information on the source spectrum (e.g. intracluster plasma temperatures) are needed to subsequently convert these primary data to other energy bands or to bolometric fluxes and luminosities.

For the calculations of the fluxes, the luminosities, and some other physical parameters in this paper we have made the following assumptions. A first approximate unabsorbed flux is calculated for each X-ray source from the observed count rate, prior to any knowledge about its nature and redshift by assuming a thermal spectrum with a temperature of $5 \mathrm{keV}$, a metallicity of 0.3 solar (with abundances taken from Anders \& Grevesse (1989). A redshift of zero, and an interstellar column density of hydrogen as obtained from Dickey \& Lockman (1990) \& Stark et al. (1992) for the X-ray source position is adopted. 
This nominal flux is used to impose the flux limit on the X-ray source sample. After a cluster has been identified and its redshift secured a better temperature estimate is obtained by means of the temperature/X-ray luminosity relation (Markevitch 1998) ${ }^{1}$, and a corrected flux and $\mathrm{X}$-ray luminosity is calculated taking the new estimated temperature, the K-correction for the observed redshift, and the dependence on the interstellar absorption into account. The X-ray luminosities are always calculated in the ROSAT band in the cluster restframe, while the fluxes are given in the ROSAT band for the observer frame as unabsorbed fluxes. The calculations are performed within the EXSAS software system (Zimmermann et al. 1994) with the spectral code from John Raymond (Raymond \& Smith 1977). Instead of using the standard codes of EXSAS for the count rate-flux conversion we are using our own macros, which have been tested against XSPEC and show a general agreement within less than $3 \%$. For the calculations of the luminosities and other physical properties of the clusters we assume a standard cosmology with $H_{0}=50 \mathrm{~km} \mathrm{~s}^{-1} \mathrm{Mpc}^{-1}, \Omega_{0}=1$ and $\Lambda=0$. While the basis of the source detections is the standard analysis of the RASS (Voges et al. 1999), we have reanalysed the source count rates and other source properties as described in Sect. 4 with the growth curve analysis technique. Note that previous comparisons of the results of this technique with deeper pointed ROSAT observations show that the measured flux underestimates the total cluster flux, typically by an amount of $7-10 \%$ (Böhringer et al. 2000). The fluxes and luminosities quoted here are the measured values without a correction for the possibly missing flux.

\section{The ROSAT All-Sky Survey data}

The starting point of the sample construction is the RASS standard analysis source list. This source list was constructed during the second RASS processing (RASS II) by Voges et al. $(1996,1999)$ using subsequently the LDETECT, MDETECT, and Maximum-likelihood algorithms (referred to as Standard Analysis Software System). While only highly significant sources (maximum likelihood parameter $L \geq 15$ ) with count rates above $0.05 \mathrm{cts} \mathrm{s}^{-1}$ and with interactively confirmed existence entered into the published RASS bright source catalogue (Voges et al. 1999), the primary, ROSAT Team internal source catalogue down to a source likelihood of $L=7$ is used here. At this low likelihood the significance of some of the sources is below $3 \sigma$ and not all of the sources are expected to be real. However, this low threshold guarantees that no sources are missed in the final sample, after the new flux cut is introduced. In total 54076 sources were found by the standard analysis in RASS II down to a likelihood of 7 in the study area of REFLEX.

\footnotetext{
${ }^{1}$ We are using the temperature/X-ray luminosity relation uncorrected for the effects of cooling flows, since the REFLEX $\mathrm{X}$-ray data used here are also not corrected for the possible effects of cooling flows.
}

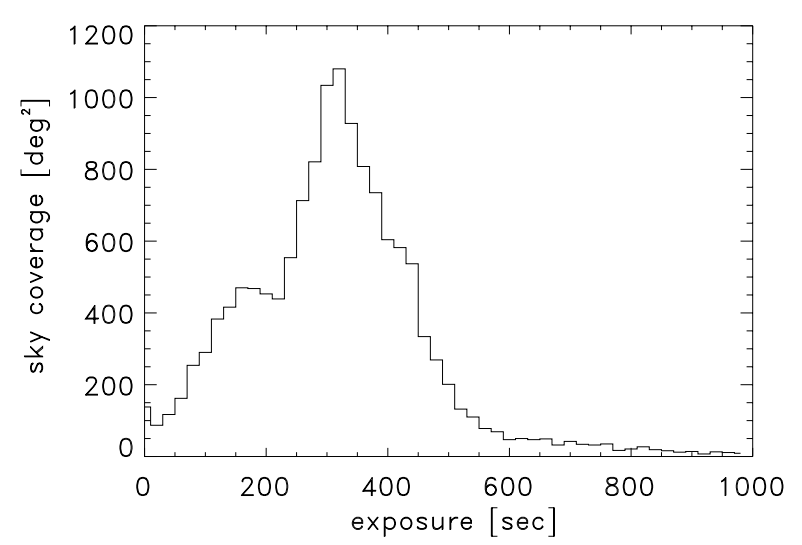

Fig. 1. Exposure time distribution of the ROSAT All-Sky Survey as analyzed in RASS II in the area of the REFLEX survey

Table 2. Fractions of the REFLEX survey area with low exposure

\begin{tabular}{lr}
\hline exposure $[\mathrm{s}]$ & fraction of the sky area \\
\hline$<50$ & 0.019 \\
$<100$ & 0.054 \\
$<150$ & 0.117 \\
$<200$ & 0.200 \\
$<300$ & 0.413 \\
\hline
\end{tabular}

One complication in using RASS data is the nonhomogeneous sky coverage. Since the sky was scanned in great circles perpendicular to the ecliptic, the shortest exposures are near the ecliptic equator, while this piles up at the ecliptic poles. In addition the satellite had to be switched-off frequently during the passages through the radiation belts. This affects in particular the southern sky data, since due to the South Atlantic Anomaly of the Earth's magnetic field the radiation belts are more prominent in the southern sky at the flight altitude of ROSAT. Some minor regions are underexposed because the data have been rejected for reasons of bad quality of the attitude solution. This leaves low exposure areas in the primary data. The resultant exposure distribution is shown in Fig. 1. The mean exposure is $335 \mathrm{~s}$ and the median is $323 \mathrm{~s}$ (compared to NORAS with mean and median exposures of 397 and $402 \mathrm{~s}$, respectively). Table 2 gives the fractions of the sky area which are underexposed. Only a few percent of the sky area are strongly underexposed and only about $12 \%$ has less than half the median exposure. Such structure imposed by the exposure drop-outs is therefore not so dramatic, but has to be taken into account for any statistical analysis of the cluster population. Its actual effects depend on the X-ray flux limit of the sample.

In particular for the clustering analysis the distribution of underexposed areas has to be known, so that it can be taken into account. This distribution is shown in Fig. 2. 


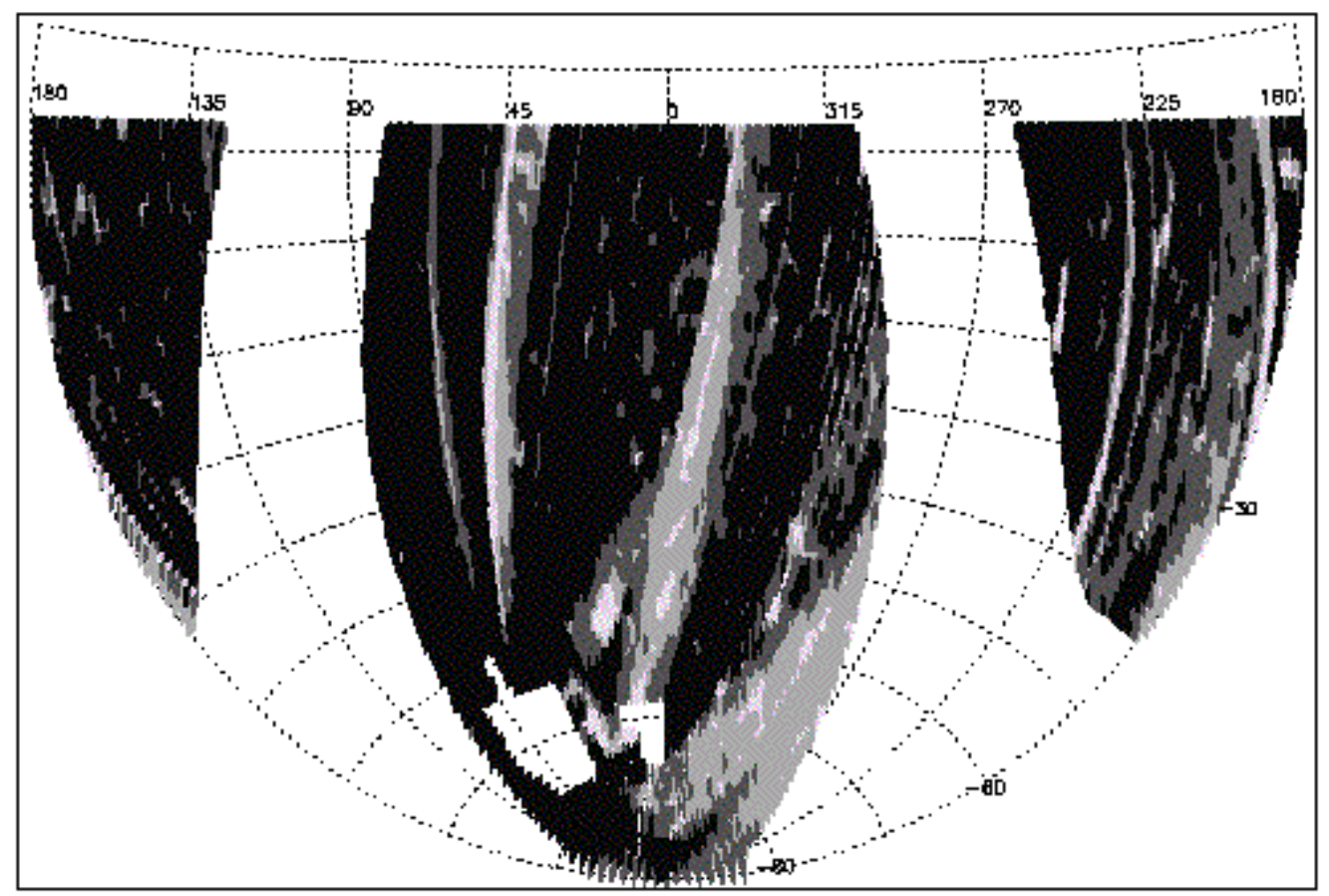

Fig. 2. Exposure distribution in the area of the REFLEX survey. Four grey levels have been used for the coding of the exposure times, with increasing intensity for $t_{\exp }<100 \mathrm{~s}, 100<t_{\exp }<200 \mathrm{~s}, 200<t_{\exp }<300 \mathrm{~s}, t_{\exp } \geq 300$, respectively. The coordinate system is equatorial for the epoch J2000

The underexposed area is not contiguous, but it is more or less confined to four strips in the southern sky. These strips reflect the shut-off times of the ROSAT-detector during the passage of the radiation belts in the South Atlantic Anomaly.

\section{Reanalysis of the X-ray data of the RASS sources with the GCA technique}

Since we found from our previous studies that the flux of extended sources is underestimated by the standard RASS source detection algorithm (Ebeling et al. 1996; De Grandi et al. 1997), a reanalysis of the source fluxes is necessary before we introduce a count rate cut or flux limit in the source list, that will subsequently serve as the basis for the construction of the X-ray flux-limited cluster sample. This is especially important in the present study since the majority of the cluster sources feature a significant extent and many appear not perfectly spherically symmetric. To this end we have developed a new source characterization technique, the growth curve analysis (GCA) method, which is described in detail in Böhringer et al. (2000). The strategy for the development of the new algorithm is to obtain reliable fluxes for extended sources and to extract as much useful information from the raw data as possible with a simple and robust technique. The simplicity of the technique is particularly important in devising a model for the source detection from theoretically constructed catalogue data in order to simulate possible selection effects in the sample. We have given preference to use the GCA method for this analysis over the methods described by Ebeling et al. (1996) and De Grandi et al. (1997) since it makes more extensive use of the photon count information and provides reliable count rates also for lower fluxes. Also the influence of assumptions made about the applied cluster model is minimized, since (as shown in Böhringer et al. 2000) almost $90 \%$ of the total flux is sampled directly with GCA and only for the final minor correction a model assumption is used. A more detailed comparison between the different methods to measure the count rate will be given elsewhere (see also Böhringer et al. 2000 for a comparison of GCA results with BCS data from Ebeling 1998). Here we give only a brief outline on the GCA.

For each source GCA returns (among other information) the following most important parameters which will be used in the source selection work:

o observed source count rate (background subtracted)

- Poisson error (photon statistics) for the count rate

- locally redetermined center of the source

- mean exposure for the source region

o significance of the source detection

o estimate of the radius out to which the source emission is significantly detected

o extrapolated source count rate (obtained by model fitting to the source emission distribution)

o hardness ratio characterizing the source spectrum and its photon statistical error

- fitted source core radius

- Kolmogorov-Smirnov test probability that the source shape is consistent with a point source

The basic parameters are derived for the photon distribution in the three energy bands "hard" (0.5 to $2.0 \mathrm{keV}$, channels 52-201), "broad" (0.1 to $2.4 \mathrm{keV}$, 
1RXS.J020144.2-021158

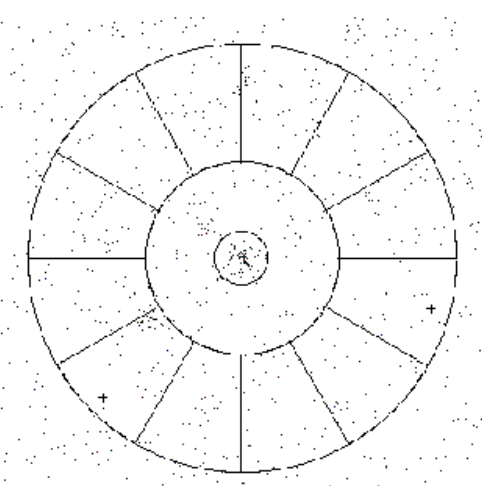

Fig. 3. Example of the set-up of the source characterization method used in the GCA technique. The image shows the hard band photon distribution from an area of the RASS in a 1.5 degree box around the X-ray source. The outer two circles enclose the area of the background determination. This background area is divided into 12 sectors. The two sectors marked by a cross are discarded from the background determination. They are flagged by a $2.3 \sigma$ clipping technique indicating a possible contamination or strong fluctuation (see Böhringer et al. 2000 for details). The inner ring marks the outer radius out to which significant X-ray emission from the source is detected

channels 11-240), and "soft" (0.1 to $0.4 \mathrm{keV}$, channel 11-40). The band definitions are the same as those used in the standard analysis (Voges et al. 1999). Here we are only using the hard band results, since the clusters are detected in this band with the highest signal to noise ratio. An exception is the hardness ratio which requires the results from the hard and soft bands.

The source count rate is determined from the cumulative, radial source count rate profile ("growth curve") after background subtraction. The construction of the growth curve is preceded by a redetermination of the source center and by the background measurement. As a typical example, the growth curve for the source shown in Fig. 3 is displayed in Fig. 4. In addition to the count rate as a function of integration radius, the uncertainty limits determined by photon statistical error (including the error for the background subtraction) also are calculated and displayed in Fig. 4 as dashed lines.

The count rate is determined in two alternative ways. In the first determination an outer radius of significant X-ray emission, $r_{\text {out }}$, is determined from the point where the increase in the $1 \sigma$ error is larger than the increase of the source signal. The integrated count rate is then taken at this radius. In the second method a horizontal level is fitted to the outer region of the growth curve (at $\left.r \geq r_{\text {out }}\right)$, and this plateau is adopted as the source flux. We use the second approach as the standard method but use also the first method as a check, and a way to estimate systematic uncertainties in the count rate determination in addition to the pure photon statistical errors. We also determine a fitted total count rate by means of a $\beta$-model as described below. For sources where close neighbours

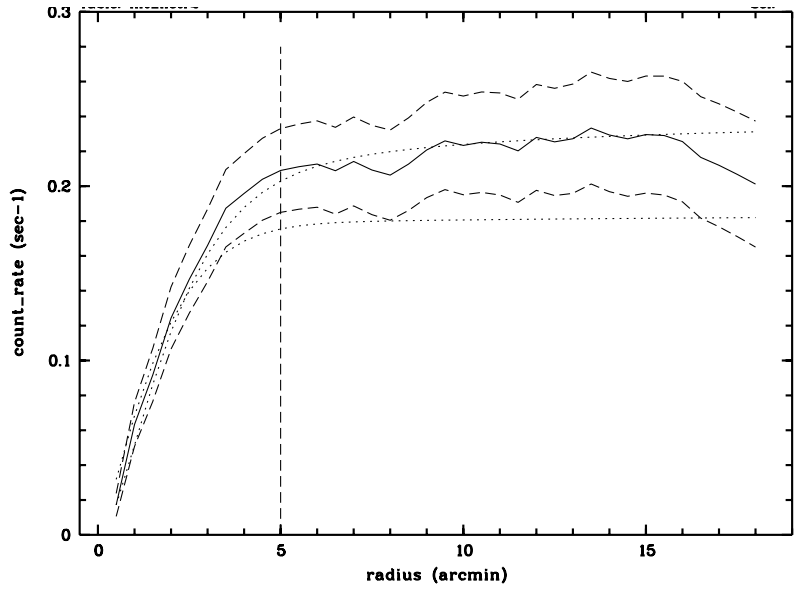

Fig. 4. Integrated count rate profile for the source shown in Fig. 3. The integrated count rate profile is background subtracted. The two dashed curves give the photon statistical error $(1 \sigma)$ of the count rate which includes the uncertainty of the signal and background determination. The vertical dashed line shows the outer source radius as explained in the text. The lower dotted line shows the $\chi^{2}$ fit of a point source to the data while the upper dotted line shows the best King model fit (including the convolution with the RASS PSF)

disturb the count rate measurement we run a separate deblending analysis.

The two most important source quality parameters determined within GCA are the spectral hardness ratio and the source extent. The hardness ratio, $H R$, is defined as $H R=\frac{H-S}{H+S}$ where $H$ is the hard band and $S$ the soft band source count rate (both determined for the same outer radius limit).

The source extent is investigated in two ways. In the first analysis a $\beta$-model profile (Cavaliere \& FuscoFemiano 1976) convolved with the averaged survey PSF (G. Hasinger, private communication) is fitted to the differential count rate profile (using a fixed value of $\beta$ of $2 / 3$ ) yielding a core radius, $r_{\mathrm{c}}$, and a fitted total count rate. Secondly, a Kolmogorov-Smirnov test is used to estimate the probability that the source is consistent with a point source. The source is flagged to be extended when the KS probability is less than 0.01. Tests with X-ray sources which have been identified with stars or AGN show a false classification rate as extended sources of about $5 \%$ (these results will be discussed in detail in a subsequent paper).

All 54076 RASS II sources in the REFLEX study region were subjected to the GCA reanalysis. All sources with a count rate $\geq 0.08 \mathrm{cts} \mathrm{s}^{-1}$ were retained for the primary sample.

For the first sample cut in count rate we have been very conservative. In addition to selecting all sources with a count rate $\geq 0.08 \mathrm{cts} \mathrm{s}^{-1}$ as measured at $r_{\text {out }}$ we have also retained all sources featuring a fitted total source count rate above this value in the $\beta$-model fit and a significance for the source detection $\geq 3 \sigma$. While this leads to the inclusion of a significant fraction of sources below the count rate cut (due to less successful $\beta$-model fits) it also 


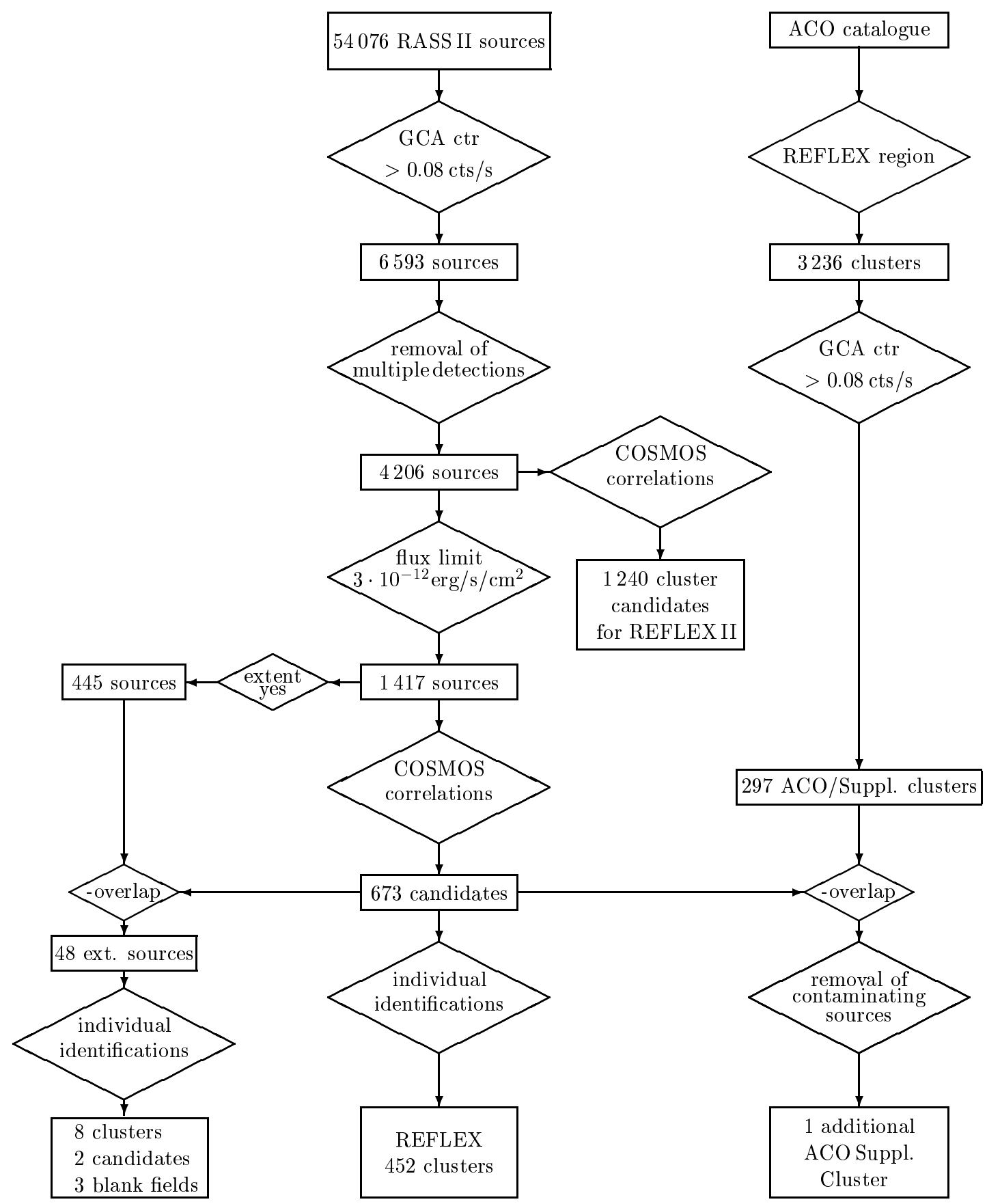

Fig. 5. Flow diagram illustrating the major data reduction steps conducted in the construction of the REFLEX sample. Also shown are two side branches of the data analysis used to test the sample completeness based on a separate search for X-ray emission in RASS II for the clusters of Abell et al. (1989) and an inspection of all extended X-ray sources in the REFLEX area above the REFLEX flux-limit

ensures that sources with pathological count rate profiles featuring an underestimate of $r_{\text {out }}$ are not lost before all sources can individually be inspected in the GCA diagnostic plots. A comparison of the GCA determined count rate (first method) and the fitted count rate is shown in Fig. 6. There is a good correlation of the two count rate values above a measured count rate of about $0.1 \mathrm{cts} \mathrm{s}^{-1}$. At low values of the GCA count rate, the fitted count rates show a large scatter. This is mostly due to the poor photon statistics providing not enough constraints on the source shape for a good enough $\beta$-model fit. A closer inspection of the results shows that at low count rates the fitted results give overestimates in more than one fourth of the sources, leading to an oversampling of about 20\%. Simulations have shown that the reason for this is an overestimate of the core radius for sources with small photon numbers. This is one reason why it is preferable not to use model fits or the SRT method described in De Grandi et al. (1997) for sources with low photon statistics. The oversampling is of no harm to the final sample 


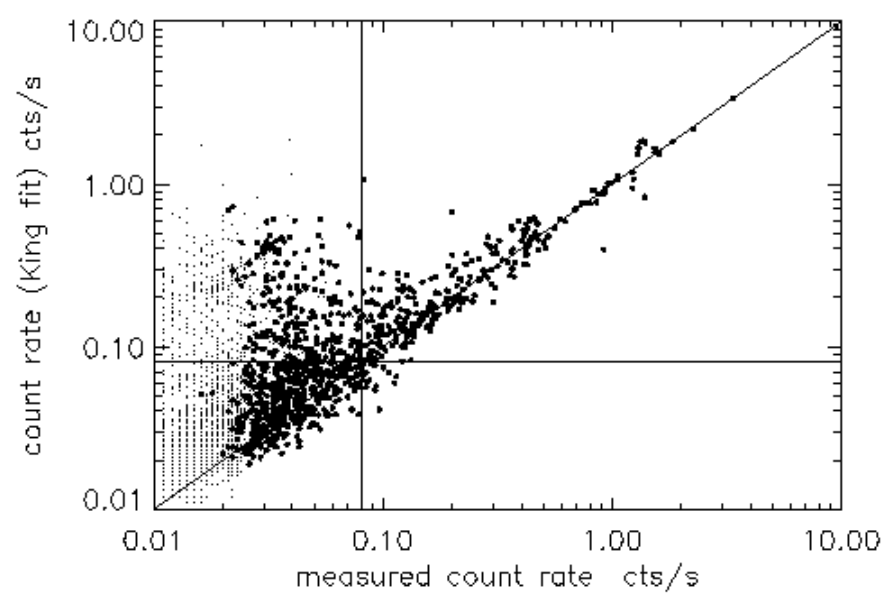

Fig. 6. Comparison of the count rates determined for the RASS sources in the REFLEX study area with both techniques, the "measured" count rate out to the radius of significant X-ray emission and the count rate obtained by fitting a King profile to the source count rate profile shape. The diagonal line gives the location of the points for which both measures are equal. The vertical and horizontal line give the count rate cut values for the two techniques, respectively. The data for which the significance of the detected signal is found to be greater than 3 are marked by heavy dots, while the data below this significance threshold are plotted by light dots. In the graph for clarity only the first 5000 sources of the total sample of 54076 sources are shown

construction, since the final REFLEX sample is obtained by another cut in flux well above this limit.

In total the first count rate cut leads to a sample of 6593 sources. This sample contains still a large number of original multiple detections of extended sources by the RASS II standard analysis. The new analysis method offers an efficient way of removing most of these multiple detections. In the redetermination of the source center in the GCA analysis, the technique usually finds a common center for the multiple detection of clusters within small numerical differences in the position (generally $<1$ arcmin). Since at a separation of 2 arcmin also point sources are already overlapping, we have used a maximal separation of 2 arcmin to identify these multiple detections as a single structure in the further identification process. Removing the redundant detections the source list shrinks to 4410 sources. This is the sample that was subjected to the first X-ray optical correlation as described in the next section. Further screening revealed another 204 redundant detections in very extended clusters where the method has settled in different local maxima (with a separation larger than 2 arcmin), but which are easily recognized visually as continuous patches in the photon distribution. Figure 5 summarizes the number of sources obtained in the subsequent steps of the X-ray source sample construction as well as the further data reduction steps described in the following.

To illustrate how well the X-ray properties can still be characterized near the flux limit of this sample we provide some statistics on the source photon number and detec-

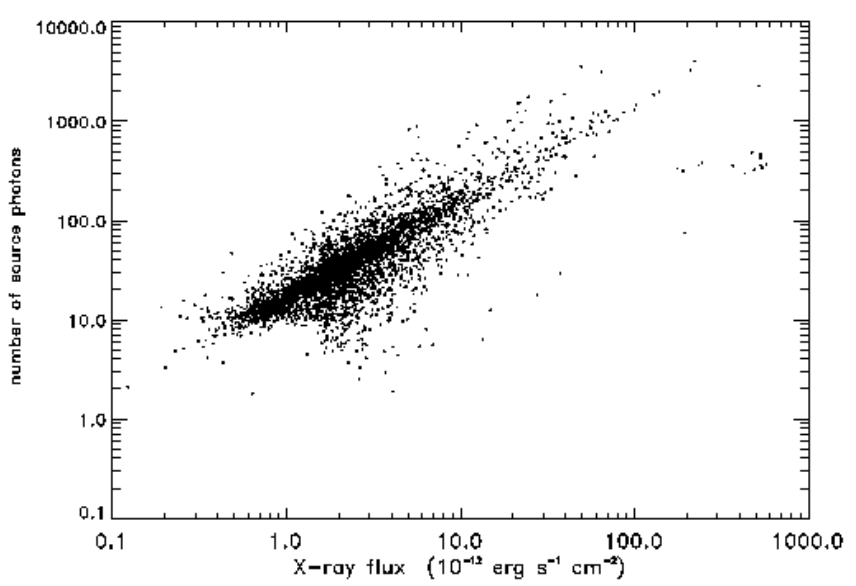

Fig. 7. Distribution of the number of source photons (background subtracted) obtained as a function of the X-ray flux

tion significance for the sources in the sample. This is interesting in the light of the discussion in Sect. 2, where we outline the strategy for the survey depth and where we argue that the depth of a survey is limited, if we require a certain accuracy for the derived X-ray properties requiring a minimum source photon number. In Fig. 7 we show the number of photons detected for the sample of 4206 RASS II sources above the count rate cut. Most sources at the count rate limit have more than 10 photons allowing for a flux determination with a formal accuracy of at least $30 \%$. Note that besides the "main sequence" of data points there is also a fraction of sources well below these typical data. These data points come from the low exposure areas in RASS II. To avoid unwanted selection effects in the sample it is useful to introduce a source count limit, and to correct for this cut in the sample selection function as discussed later.

Figure 8 shows in a similar way the typical signalto-noise ratio $\left(S / N=N_{\mathrm{s}} / \sqrt{N_{\mathrm{s}}+N_{\mathrm{b}}}\right.$, where $N_{\mathrm{s}}$ are the source counts and $N_{\mathrm{b}}$ are the background counts in the source region) for the flux measurement of a source as a function of the X-ray flux. Again there are some sources with a low significance for the source flux determination, which come from the low exposure areas.

\section{Correlation of the $\mathrm{X}$-ray source list with the COSMOS data base}

\subsection{The COSMOS data base}

Since the X-ray properties which are described above do not allow by themselves an identification of the X-ray sources associated to clusters, we have to include information from an optical data base in the further identification process. For this we are using the most comprehensive optical data base covering the southern sky and the area of the REFLEX survey: the COSMOS scans of the UK-Schmidt survey plates (MacGillivray \& Stobie 1984). There are also the complementary APM scans of the same photographic survey material, but the galaxy classification in the APM survey concentrates on the southern part of 


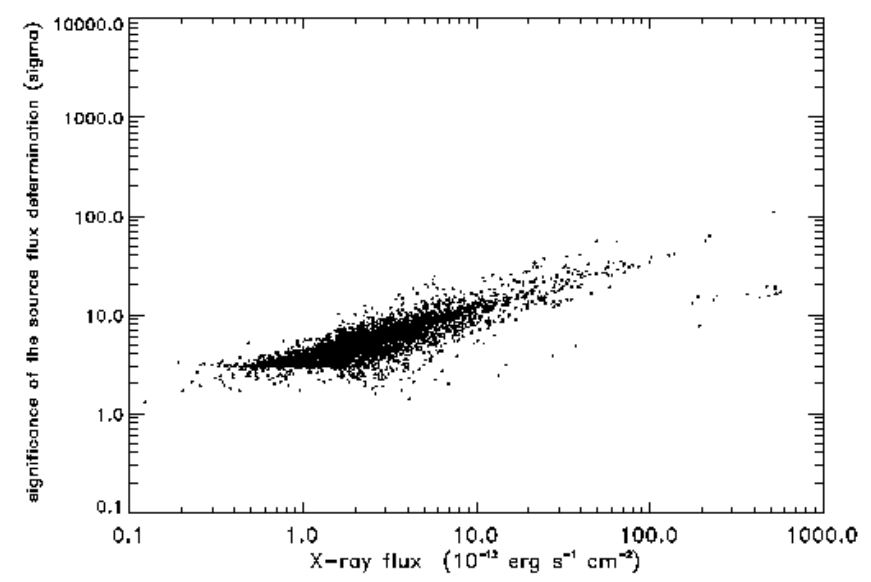

Fig. 8. Distribution of the signal-to-noise of the flux determination as a function of the X-ray flux. For the definition of the significance parameter see the text

the sky south of the galactic plane (Maddox et al. 1990) which covers only about $2 / 3$ of the REFLEX region.

The UK-Schmidt survey has been performed using IIIa-J photographic plates at the $1.2 \mathrm{~m}$ UK-Schmidttelescope. The plates were scanned within a sky area of about $5.35 \mathrm{deg} \times 5.35 \mathrm{deg}$ per plate with the fast COSMOS scanning machine and subsequently analysed yielding 32 parameters for the source characterization per object. These parameters describe the object position, intensity, shape, and classify the type of object. Object images are recognized down to about $b_{\mathrm{J}} \sim 22 \mathrm{mag}$. This allows a subsequent star/galaxy separation which has been estimated to be about $95 \%$ complete with about $5 \%$ contamination to $b_{\mathrm{J}} \sim 19.5 \mathrm{mag}$ and about $90 \%$ complete with about $10 \%$ contamination to $b_{\mathrm{J}} \sim 20.5 \mathrm{mag}$ (Heydon-Dumbleton et al. 1989; Yentis et al. 1992; MacGillivray et al. 1994, and Mac Gillivray priv. communication). The galaxy magnitudes were intercalibrated between the different plates using the substantial plate overlaps and absolutely calibrated by CCD sequences (Heydon-Dumbleton et al. 1989; MacGillivray et al. 1994).

\subsection{Correlation of the $X$-ray sources with the COSMOS galaxy distribution}

In search of cluster candidates as counterparts to the RASS sources we correlate the X-ray source positions with the galaxy catalogue of the COSMOS data base. The basis of this correlation are counts of galaxies in circles around the X-ray source positions to search for galaxy density enhancements.

Here we should make some remarks about the strategy behind the choice of the present cluster search algorithm. As mentioned before it is difficult to devise a good algorithm to select the most massive clusters of galaxies from optical sky survey images. We use a comparatively simple algorithm (aperture counts as compared to e.g. matched filter techniques). This simple technique seems well adapted to our needs and the depth of the COSMOS data set: (i) the technique is used to only flag the candidates and there is no need to determine a cluster richness, since we use the X-ray emission for a quantitative measure of the clusters; (ii) while matched filter techniques may introduce a bias, since a priori assumptions are made about the shape of an idealized, azimuthally symmetric cluster, we are interested in introducing as little bias and as few presumptions as possible; (iii) the actual numbers in the galaxy counts are limited and therefore the shape matching is not precise and is affected by low number statistical noise. Therefore our technique is not seen as a perfect and objective cluster characterization algorithm. The cluster selection should primarily depend on the $\mathrm{X}$-ray criteria. We have chosen a very low cut for the optical selection which results in a substantially larger candidate sample compared to the expected number of clusters, with an estimated contamination of as much as $30-40 \%$. But it assures on the other hand that we have a highly complete candidate sample. This overabundance of candidates is thus a necessary condition to obtain an essentially $\mathrm{X}$-ray selected sample for our survey.

The galaxy counts are performed for 5 different radial aperture sizes: $1.5,3,5,7.5$, and 10 arcmin radius with no magnitude limit for the galaxies selected. Since an aperture size of about $0.5 h_{50}^{-1} \mathrm{Mpc}$ in physical scale corresponding to about two core radii of a rich cluster provides a good sampling of the high signal-to-noise part of the galaxy overdensity in a cluster, the chosen set of apertures gives a good redshift coverage in the range from about $z=0.02$ to 0.3 as shown by the values given in Table 4 . With this choice and the depth limit of the COSMOS data set we are aiming at a high completeness in the cluster search out to a redshift of about $z=0.3$. For this goal the chosen flux limit and the depth of the COSMOS data base are quite well matched as the richest and most massive clusters are still detected in both data sets out to this redshift.

The galaxy counts around the given X-ray source positions are compared with the number count distributions for 1000 random positions for each photographic plate. With this comparison we are also accounting for plate to plate variations in depth as explained below. The number count histograms for the random positions have been generated at the Naval Research Laboratory in preparation of a COSMOS galaxy cluster catalogue, the SGP pilot study (Yentis et al. 1992; Cruddace et al. 2000), and for this ESO key program. The results of the random counts yield a differential probability density distribution, $\phi\left(N_{\text {gal }}\right)$, of finding a number of $N_{\text {gal }}$ galaxies at random positions. An example for the distribution $\phi\left(N_{\text {gal }}\right)$ for an average of 5 randomly selected plates is shown in Fig. 9 for all five aperture sizes. (Note that $\phi\left(N_{\text {gal }}\right)$ is defined here as a normalized probability density distribution function while in Fig. 9 we show histograms of the form $\left.\phi\left(N_{\text {gal }}\right) \times N_{\text {count }}\right)$. The distribution functions resemble Poisson distributions (The possible theoretical description of the functions is not further pursued here since we are only interested in the purely empirical application to the following 


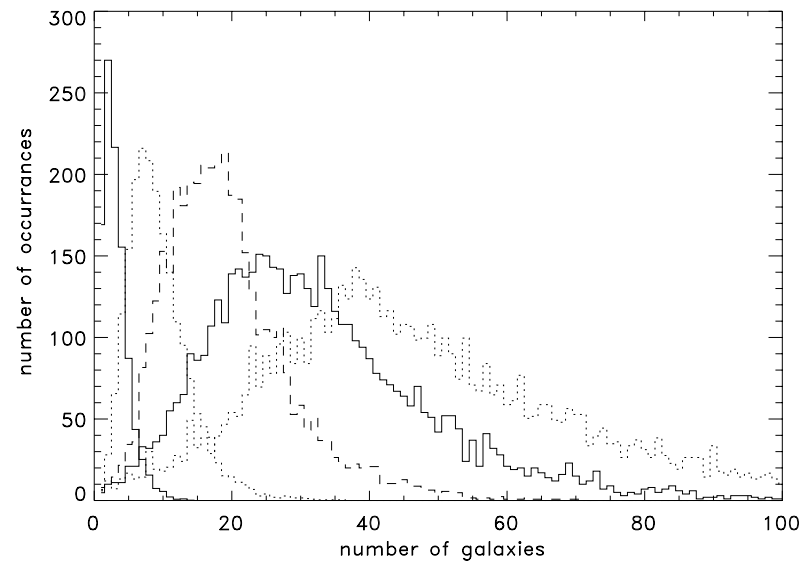

Fig. 9. Example of the distributions of galaxy number counts, $\phi\left(N_{\text {gal }}\right) \times N_{\text {count }}$, for the five apertures with radii of $1.5,3$, $5,7.5$, and 10 arcmin. The histograms are constructed from counts at 1000 random positions per photographic plate and the results for each aperture size as shown here are obtained from an average of five plates. The second, third, forth, and fifth histograms have been multiplied by factors of $2,4,5$, and 6 , respectively, for easier comparison

statistical analysis). In Fig. 10 the random count histogram for aperture 2 ( 3 arcmin radius) is compared to the counting results for the $4206 \mathrm{X}$-ray source positions. We note the large number of sources with significant galaxy overdensities in the X-ray source sample compared to the random counts, and expect to find the X-ray clusters in this high count tail of the distribution.

These results for $\phi\left(N_{\text {gal }}\right)$ are then used in the form of cumulative probability distribution functions

$P\left(<N_{\text {gal }}\right)=\int_{0}^{N_{\text {gal }}} \phi\left(N_{\text {gal }}^{\prime}\right) \mathrm{d} N_{\text {gal }}^{\prime}$

to assign the probability value $P\left(<N_{\text {gal }}\right)$ to each counting result.

For the counts around X-ray sources we expect a significant galaxy density enhancement for those sources which have cluster counterparts. Therefore the counting results for the X-ray source positions should yield a distribution function $\phi\left(N_{\text {gal }}\right)_{\mathrm{X}}$ which has a more pronounced tail at high values of $N_{\text {gal }}$ (Fig. 10). Instead of characterizing the enhancement of the counts at high galaxy numbers in the tail of $\phi\left(N_{\text {gal }}\right)$ X we use another data representation as follows.

Going back to the random sample, taking each of the values of $P\left(<N_{\text {gal }}\right)$ assigned to each counting result, and plotting the distribution function $\phi\left(P\left(<N_{\text {gal }}\right)\right) \equiv$ $\phi(P(N))$ we will find that this function is a constant. This follows simply from the chain rule of differentiation in the following way

$\phi(P(N)) \mathrm{d} P=\phi\left(N_{\text {gal }}\right)\left|\frac{\mathrm{d} N_{\text {gal }}}{\mathrm{d} P}\right| \mathrm{d} P$

$\left.=\phi\left(N_{\text {gal }}\right)\left(\frac{\mathrm{d}}{\mathrm{d} N_{\text {gal }}} \int_{0}^{N_{\text {gal }}} \phi\left(N_{\text {gal }}^{\prime}\right) \mathrm{d} N_{\text {gal }}^{\prime}\right)\right)^{-1} \mathrm{~d} P=$ const.

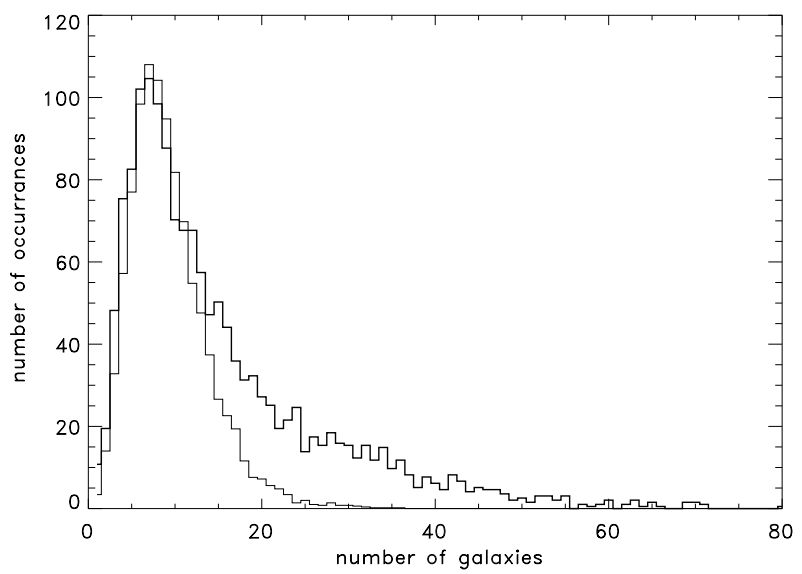

Fig. 10. Example of the distributions of galaxy number counts in a circular aperture with 3 arcmin radius for an average of five UK Schmidt plates and 1000 random positions per plate (thin line). This distribution is compared to the results of the galaxy number counts for the 4206 X-ray sources of the sample for the same aperture radius. The histogram for the random position counts has been normalized to the histogram of the $\mathrm{X}$-ray source counts so that the peaks have the same hight

Thus for random counts we should expect to see a constant function (with noise if the counts are derived in an experiment independent from the random count experiment used to define $\left.P\left(<N_{\text {gal }}\right)\right)$. In the case of counts around $\mathrm{X}$-ray sources involving clusters of galaxies the function $\phi\left(P_{\mathrm{X}}(N)\right)$ is no longer a constant but should show an enhancement for large values of $P_{\mathrm{X}}(N)$. Figure 11 shows the resulting distribution function for the galaxy counts, $\phi\left(P_{\mathrm{X}}(N)\right) \times N_{\text {sources }}$, in the 3 arcmin ring aperture for the sample of 4206 X-ray sources. The extended tail in the distribution at high values of $N_{\text {gal }}$ in Fig. 10 now translates into a very pronounced peak at large values of $P$ in Fig. 11.

For the further evaluation of this type of diagrams we make the following simplifying assumptions: i) the distribution function is composed of two types of counting results, results obtained for cluster X-ray sources and results obtained for other sources, and ii) the non-cluster $\mathrm{X}$-ray sources are not correlated to the galaxy distribution in the COSMOS data base and thus constitute effectively a set of random counts. This latter assumption is of course not strictly true for all the non-cluster X-ray sources. While it may be justified to treat stars and other galactic sources as well as distant quasars as independent of the nearby galaxy distribution, there is also a population of extragalactic sources like low redshift AGN and starburst-galaxies that we know are correlated to the large-scale structure in the galaxy distribution. However, the practical assumption that this correlation is weak in comparison to the galaxy density enhancements in clusters of galaxies is generally well justified.

With this assumption we expect to find a distribution function $\phi\left(P_{\mathrm{X}}(N)\right)$ composed of a constant function and a peak at high $P$-values. Subtracting the constant function leaves us with the cluster sources. This is schematically 


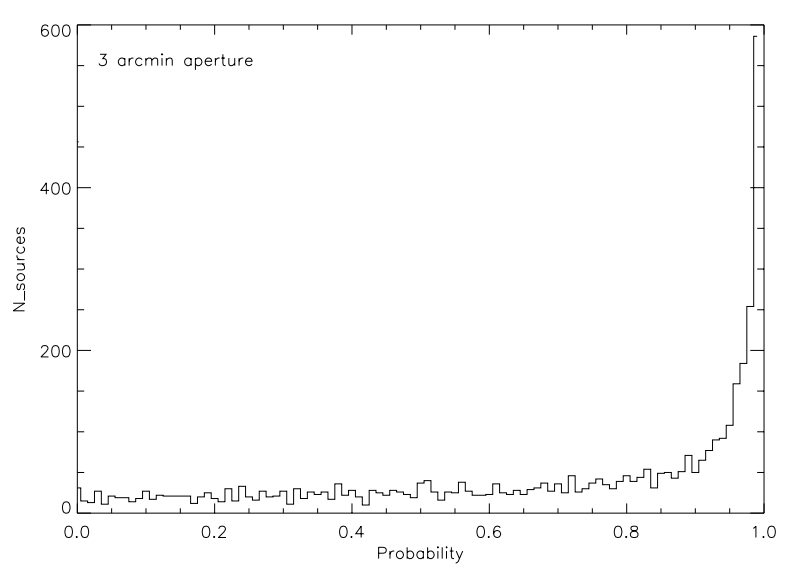

Fig. 11. Histogram of the galaxy excess probabilities, $P_{\mathrm{X}}$, obtained from galaxy counts in circular apertures of radius 3 arcmin for the sample of 4206 sources of our count rate limited sample of southern RASS II sources (excluding the multiple detections of extended sources). There is a clear excess of counts at high values of the galaxy density (high values of $P_{\mathrm{X}}$ ), which is primarily due to the effect of galaxy cluster counterparts to the X-ray sources

illustrated in Fig. 12. For the selection of the cluster candidates we can now either select the sources which feature a high value of $N_{\text {gal }}$ or a high value of $P_{\mathrm{X}}(N)$. We choose to use $P_{\mathrm{X}}(N)$ for the sample selection (as justified further below) in such a way that most of the cluster peak is included in the extracted sample (that is choosing $P_{\mathrm{X}}(N)$ such that the fraction $C$ in Fig. 12 of cluster lost from the sample is small or negligible).

The clear distinction between the flat distribution for probability values between 0 and about 0.7 and the clear and prominent "cluster peak" as found in Fig. 11 indicates that we can quantify this result further. As illustrated in the sketch of Fig. 12, the cluster contribution is responsible for the dark shaded areas labeled $A$ and $C$. Extracting a sample highly enriched in clusters by choosing a particular high value, $P_{\mathrm{X}}^{\star}$, leaves us with a formal completeness of the sample expressed by

$F_{\text {comp }}=\frac{A}{A+C}$.

The formal contamination of this sample by non-cluster sources is likewise given by the expression

$F_{\text {cont }}=\frac{B}{A+B}$

We choose the parameter $P_{\mathrm{X}}(N)$ for the selection of the cluster sample for the following reason. The distribution $\phi\left(N_{\text {gal }}\right)$ is computed for each plate. Since there are plate to plate variations in the average galaxy density, using just $N_{\text {gal }}$ would introduce a bias in the sample extraction. The use of the parameter $P_{\mathrm{X}}(N)$ takes these variations into account. Possible variations in the background density of the galaxies within each plate are not accounted for in this approach but in general these variations are very small and due to our strategy of oversampling (minimizing fraction $C$ in Fig. 11) this has little effect on the sample.

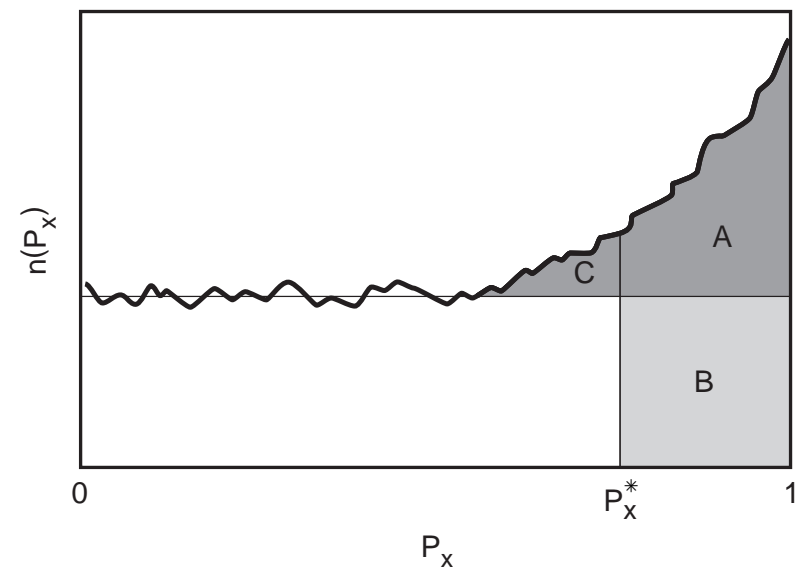

Fig. 12. Sketch of the typical result of the distribution function $P_{\mathrm{X}}\left(N_{\text {gal }}\right)$ for an X-ray source sample containing galaxy cluster counterparts. The parameter $P_{\mathrm{X}}^{\star}$ indicates the minimal allowed value of $P_{\mathrm{X}}\left(N_{\text {gal }}\right)$ in the sample selection. $A+C$ gives the "true number of clusters" and $A+B$ the size of the extracted sample

The analysis was carried out for all five circular apertures. The strategy for the selection of the cut value, $P_{\mathrm{X}}^{\star}$, was to roughly obtain a sample with $90 \%$ completeness for the single ring statistics and a contamination not much larger than about $20 \%$ to $30 \%$. Comparing the results for different apertures, one notes that the peak is best defined for the counts with the two smallest apertures. With increasing aperture size the peaks get broader and broader, leading to a more and more unfavorable value for completeness versus contamination result. Therefore we have relaxed the completeness criterion for the three largest apertures to values lower than $90 \%$ not to increase the sample contamination dramatically. The resulting values for $P_{\mathrm{X}}^{\star}, F_{\text {comp }}, F_{\text {cont }}$, and the resulting sample sizes are given in Table 4 for each aperture counting result. We also indicate in the table the sample size, $N_{\text {sample, defined as }}$ $A+B$, and the expected number of clusters, $N_{\text {cl.est., given }}$ by $A+C$ (in Fig. 12). Note that the sample size is larger than half of the starting sample (4410 objects) and that the results indicate the presence of roughly 1800 galaxy clusters, a number much larger than expected.

\section{Inspection of the first selected candidate sample}

A major reason for the large number of cluster candidates found in the above described selection process is easily found by an inspection of the optical images of the selected candidates. The main contribution of spurious clusters comes from bright stars $\left(m_{\mathrm{b}}<12 \mathrm{mag}\right)$ and nearby galaxies. For the bright stars the diffraction spikes visible on the optical plates are often split up by the object detection algorithm of COSMOS into a string of single objects mainly classified as galaxies. Therefore these bright stars appear in the statistics as "clusters of galaxies". Similarly some nearby galaxies are split up into multiple objects. Both cases are trivially recognized in a first 
Table 3. Statistics of the results of the galaxy counts around the 4410 X-ray sources (above the first count rate cut). Columns 2 to 5 give the lower probability limit for the sample selection, the sample completeness, the contamination, the sample size and the estimated total number of clusters (see text for more details). Column 6 gives the physical scale of the aperture radius at a redshift $z=0.08$, close to the median redshift of the REFLEX sample. Column 7 gives the redshift at which the aperture radius corresponds to a physical size of $0.5 h_{50}^{-1} \mathrm{Mpc}$. The combined sample is defined by all candidates flagged in at least one of the aperture count searches

\begin{tabular}{llrlrrrr}
\hline aperture radius & $P_{\mathrm{X}}^{\star}$ & $F_{\text {comp }}$ & $F_{\text {cont }}$ & $N_{\text {sample }}$ & $N_{\text {cl.est. }}$ radius $(\mathrm{Mpc})$ & redshift for $r=0.5 \mathrm{MPC}$ \\
\hline 1.5 arcmin & 0.84 & $90 \%$ & $19 \%$ & 2003 & 1808 & 0.19 & 0.305 \\
3.0 arcmin & 0.84 & $90 \%$ & $19 \%$ & 2146 & 1930 & 0.37 & 0.12 \\
5.0 arcmin & 0.90 & $75 \%$ & $18 \%$ & 1582 & 1725 & 0.61 & 0.06 \\
7.5 arcmin & 0.91 & $60 \%$ & $23 \%$ & 1224 & 1568 & 0.92 & 0.045 \\
10. arcmin & 0.91 & $50 \%$ & $30 \%$ & 1027 & 1432 & 1.23 & 0.03 \\
comb.sample & - & $>90 \%$ & - & 2640 & $\sim 1750$ & & \\
\hline
\end{tabular}

inspection of the optical fields around the X-ray sources on the POSS and UK Schmidt plates. They can therefore be easily removed from the sample. Another class of sources enhancing the cluster peak in the statistics given in Table 3 are multiple detections of very extended X-ray cluster sources. In these sources the multiple detections have larger separations than two arcmin (see Sect. 4) generally because the center determination settled on local maxima or photon density fluctuations. They are also easy to remove by an inspection of the photon distribution in the source fields. We have removed redundant detections for all sources were multiple detections occured within a well connected diffuse source photon distribution.

In total 452 stars with clearly visible diffraction spikes, 32 nearby galaxies, and 204 redundant detections of diffuse $\mathrm{X}$-ray sources were removed from the sample. With the cleaned sample we can now repeat the statistical analysis with results given in Table 4 . The sample selection cut is kept the same as above. (Note that in this statistics there are about $8 \%$ of the sources missing which leads to a lower normalization but has no effect on the conclusions drawn in the following). We note that this time the statistics indicates a number of about 800-900 for the expected number of clusters in the sample which is close to our expectations based on a comparison with cluster number counts found in deeper surveys (e.g. Gioia et al. 1990; Rosati 1998) as discussed in Sect. 2. We also note, that for the combined sample of candidates from the different aperture sizes, we obtain a total sample size which is about a factor of $\sim 1.5$ larger than the estimated number of true clusters. Thus we expect a level of contamination of non-cluster sources around $30-40 \%$. This implies a laborious further identification work to clean the sample from the contamination, a price to be paid for the high completeness level aimed for.

\subsection{Comparison of the cluster search statistics with the final results of the REFLEX Survey}

To analyse how well the cluster selection has worked we anticipate the results of the REFLEX survey and the fi- nal identification of the cluster candidates. We repeat the statistical analysis including all the sources from the starting sample with a flux in excess of $310^{-12} \mathrm{erg} \mathrm{s}^{-1} \mathrm{~cm}^{-2}$, corresponding to the flux limit of the REFLEX sample (1417 sources without the multiple detections) except for the stars with diffraction spikes, and the nearby galaxies (1169 X-ray sources). The results of the cluster search for this high-flux sample (with the same values for the selection parameter, $P_{\mathrm{X}}^{\star}$ as used before) and the comparison with the final REFLEX sample is given in Table 5 .

We note from the results given in Table 5 that the predictions are very close to the actual findings. One has to be careful, however, in the interpretation of this comparison. In fact the general agreement should not be surprising as we have used the same statistics to select the sample and we have not yet used any independent means to include clusters missed by our search to check the incompleteness independently. Nevertheless a few results are striking. The number of clusters predicted to be found is close to the number actually identified. This shows that the signal observed in the diagnostic plots of the type of Fig. 12 is indeed due to galaxy clusters and there is no large contamination by other objects. Had we found for example much less clusters than predicted, we would be forced to speculate on the presence of another source population that mimics clusters in our analysis. This is obviously not the case and the high $P_{\mathrm{X}}(N)$ signal is correctly representing the clusters in the REFLEX sample. Also the trend in the efficiency of the different apertures in finding the clusters is predicted roughly correctly. There are only small differences, as for example that the total number of clusters and the contamination predicted from the results of the first two apertures are too high and too low, respectively.

Most of the clusters identified in the REFLEX survey $(96 \%)$ are detected in the search with aperture 2 with a radius of 3 arcmin. That this ring size is the most effective is also shown in Fig. 13 where we compare the distribution of the probability values $P_{\mathrm{X}}(N)$ for the source sample and for the subsample which was identified as clusters in the course of the REFLEX Survey. Only 16 additional clusters are found in aperture 1 with 1.5 arcmin radius and 
Table 4. Statistics of the results of the galaxy counts around the X-ray sources in the sample cleaned from bright stars and multiple detections. The columns are as in Table 4

\begin{tabular}{llllrr}
\hline aperture radius & $P_{\mathrm{X}}^{\star}$ & $F_{\text {comp }}$ & $F_{\text {cont }}$ & $N_{\text {sample }}$ & $N_{\text {cl.est. }}$ \\
\hline 1.5 arcmin & 0.84 & $88.5 \%$ & $22 \%$ & 974 & 863 \\
3.0 arcmin & 0.84 & $90 \%$ & $24 \%$ & 890 & 836 \\
5.0 arcmin & 0.90 & $82 \%$ & $21 \%$ & 703 & 767 \\
7.5 arcmin & 0.91 & $65 \%$ & $26 \%$ & 606 & 718 \\
10. arcmin & 0.91 & $52 \%$ & $32 \%$ & 562 & 690 \\
comb.sample & - & $>90 \%$ & $30-40 \%$ & 1240 & sim850 \\
\hline
\end{tabular}

Table 5. Cluster search statistics for X-ray sources above a flux limit of $310^{-12} \mathrm{erg} \mathrm{s}^{-1} \mathrm{~cm}^{-2}$ and comparison with the final REFLEX sample. The first 6 columns are as in Table 4. Column 7 gives the number of REFLEX cluster detected by the specific aperture search, Col. 8 the fraction detected compared to the total REFLEX sample, and Col. 9 the contamination fraction found in the candidate sample selected by the specific aperture

\begin{tabular}{|c|c|c|c|c|c|c|c|c|}
\hline aperture radius & $P_{\mathrm{X}}^{\star}$ & $F_{\text {comp }}$ & $F_{\text {cont }}$ & $N_{\text {sample }}$ & $N_{\text {cl.est. }}$ & clusters found & $F_{\text {det }}$ & $F_{\text {cont* }}$ \\
\hline $1.5 \operatorname{arcmin}$ & 0.84 & $91 \%$ & $18 \%$ & 580 & 530 & 411 & $90 \%$ & $29 \%$ \\
\hline $3.0 \operatorname{arcmin}$ & 0.84 & $93 \%$ & $17 \%$ & 586 & 516 & 433 & $96 \%$ & $26 \%$ \\
\hline 5.0 arcmin & 0.90 & $87 \%$ & $16 \%$ & 487 & 470 & 401 & $89 \%$ & $18 \%$ \\
\hline 7.5 arcmin & 0.91 & $75 \%$ & $19 \%$ & 406 & 448 & 341 & $75 \%$ & $16 \%$ \\
\hline 10. $\operatorname{arcmin}$ & 0.91 & $64 \%$ & $22 \%$ & 354 & 438 & 299 & $66 \%$ & $16 \%$ \\
\hline comb.sample & & $\sim 95 \%$ & $30-40 \%$ & 673 & $\sim 500$ & & & $33 \%$ \\
\hline
\end{tabular}

only 3 in the last three apertures. The peak of the cluster signal is less constrained in the apertures $1,3,4$, and 5 as shown in Fig. 13. The large overlap in the detection of the clusters with the different apertures is illustrated in Fig. 14 for apertures 2, 3, and 4. Compared to the statistics in the starting sample the predicted completeness has increased for the high flux sample mostly due to the fact that the cluster signal in the statistical analysis becomes better defined with increasing flux limit. Thus the statistics of aperture 2 alone gives an internal completeness estimate of $93 \%$. Since the results of the different searches are highly correlated (see Fig. 14) we cannot easily combine the results in a statistically strict sense. A rough estimate is given by a simple extrapolation from the completeness and the sample size found for aperture 2 (93\% for 433 clusters) and the additional number of 19 clusters found exclusively in other rings yielding a formal value of $97 \%$.

The latter number should be treated with care, however, as an internal completeness check. This statistics would be more reliable if we had one homogeneous population of clusters. Since our clusters cover a wide range of richnesses and redshifts we cannot assume that all subsamples contribute to the cluster signal in Fig. 12 in the same way. If for example no significant galaxy overdensity could be detected for the high redshift clusters, this subsample would not enter into the statistics at all. Likewise, galaxy clusters for which the X-ray detections are missed in the basic source detection process are also not included in this prediction. Therefore the good agreement between the above predictions and the final results supports our

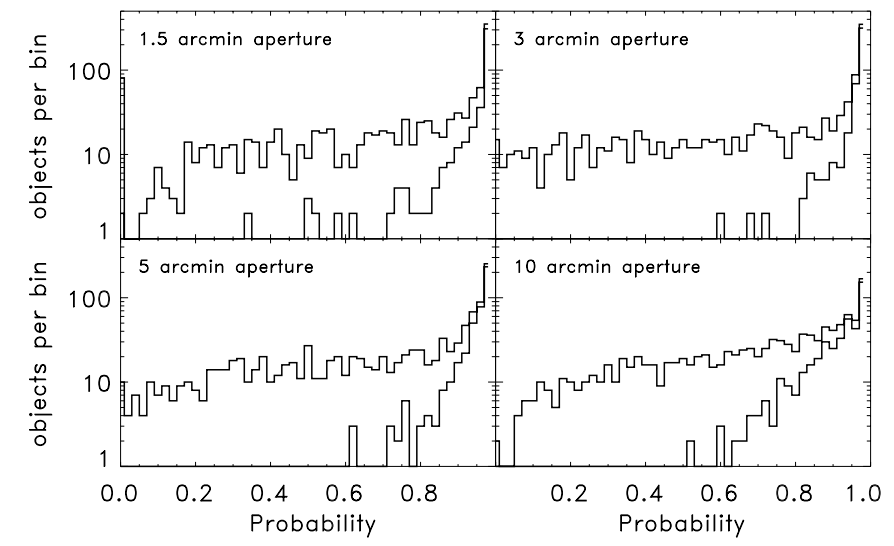

Fig. 13. Results for the cluster search with four of the five different circular apertures for the flux limit of the REFLEX sample. The thin (upper) line shows the statistics for the input sample and the thick (lower) line the results for the REFLEX clusters. The probability values plotted are defined by Eq. (1)

confidence in the high quality of the sample but it is not a sufficient test for completeness. We discuss further external tests in Sect. 8.

Another useful illustration concerns the question of how well defined the galaxy overdensity signal is for an individual cluster. An answer is given in Figs. 15 and 16 where we show the number of galaxies (above the background density) for the galaxy counts in aperture 1, 2, and 3 for the clusters of the REFLEX sample. For aperture 2 we find for example that the typical count result is about 20 galaxies per cluster providing a signal of about $4 \sigma$. Thus in general the overdensity signal is very well defined. 


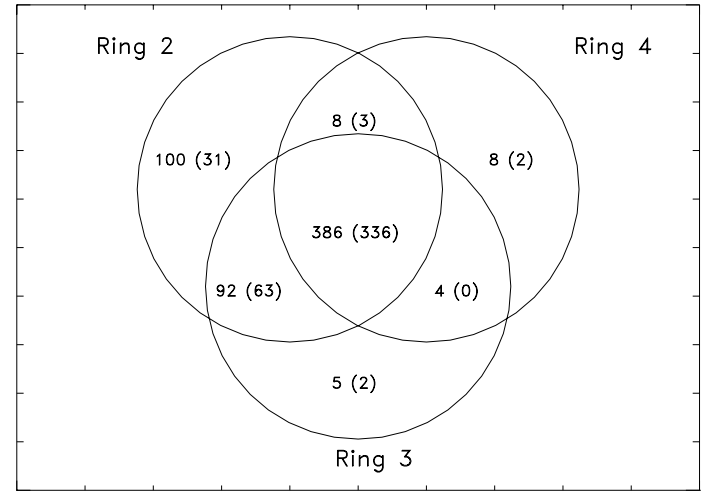

Fig. 14. Number of cluster candidates selected by means of the aperture counts in rings 1 to 3 (3, 5, 7.5 arcmin, respectively) and number of clusters found in the REFLEX Survey (values in brackets)

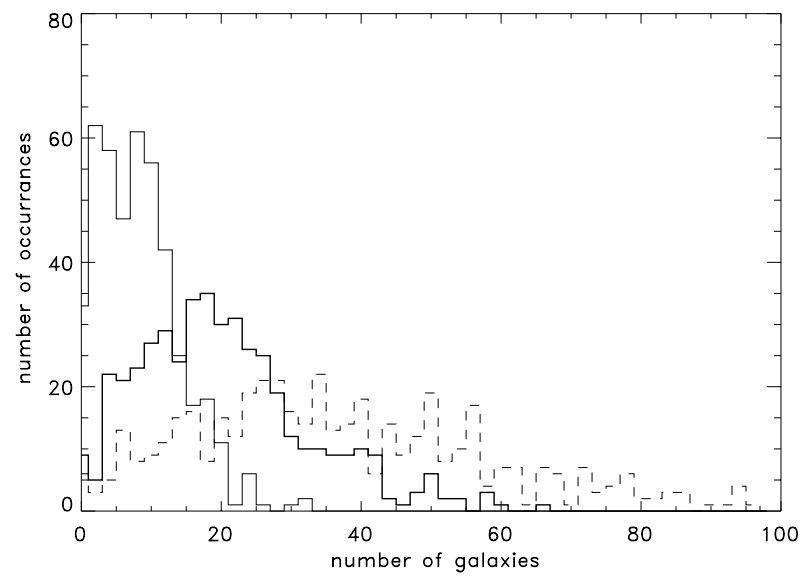

Fig. 15. Distribution of the numbers of galaxies detected in rings $1-3$ for the clusters in the REFLEX sample. Thin line: 1.5 arcmin aperture, thick line: 3 arcmin aperture, broken line: 5 arcmin aperture

There is a tail to low number counts and significances which involves only a few clusters, however. For aperture 1 we note that the number counts and significance values are substantially less. Increasing the aperture size beyond 3 arcmin increases the mean significance of the galaxy counts, as seen in the results for aperture 3 . But what is more important: the tail towards low significance values is not reduced if we compare aperture 3 to aperture 2 . This once again shows the effectiveness of aperture 2 . One should also note the strong overlap of the results of the different apertures as illustrated in Fig. 14 which is reenforcing the significance of the selection results. In fact, about $60 \%$ of all the REFLEX clusters are flagged in the counting results for all five apertures. It is not surprising that the second aperture with a 3 arcmin radius features as best adapted for our survey, since 3 arcmin corresponds to a physical scale of about $370 h_{50}^{-1} \mathrm{kpc}$ at the median distance of the REFLEX clusters, $z \sim 0.08$, (see also Table 3 ). This corresponds to about 1.5 core radii, a good sample radius to capture the high surface density part of the clusters.

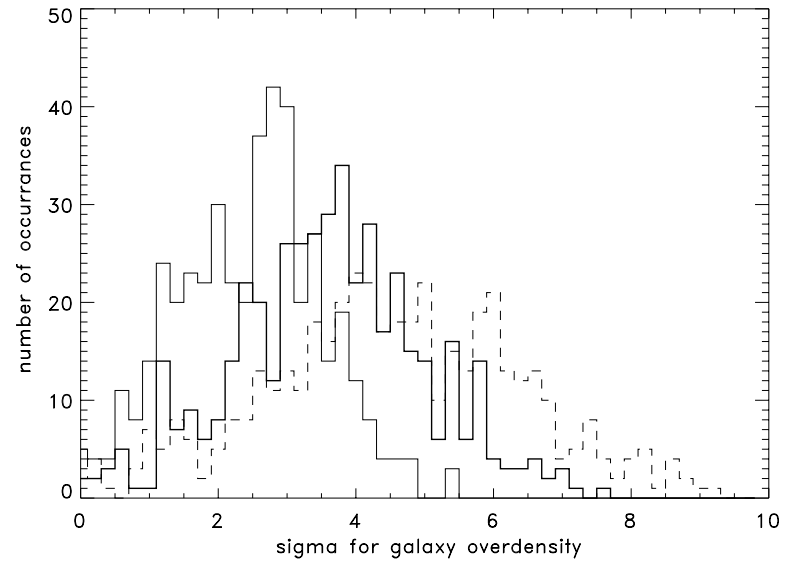

Fig. 16. Distribution of the significance values of the detections of galaxy overdensities in rings $1-3$ for the clusters in the REFLEX sample

In Figs. 17 to 19 we show the galaxy number counts and significances for aperture 2 as a function of flux and redshift. While there is no striking correlation with flux we clearly note the decrease of the number counts and significance values even for the richest clusters with redshift. In Fig. 18 we also show the expected number counts for a rich cluster (with an Abell richness of 100, which is the number of galaxies within $r=3 h_{50}^{-1} \mathrm{Mpc}$ and a magnitude interval ranging from the third brightest galaxy to a limit 2 magnitudes deeper) as a function of redshift for three magnitude limits for the galaxy detection on the plates of $b_{\mathrm{J}}=20$, 21, 22 mag. Galaxies are still classified in the COSMOS data base down to 22nd magnitude but the completeness is decreasing continuously over the magnitude range from $b_{\mathrm{J}}=20-22$. For the calculation we assume a Schechter function for the galaxy luminosity function with a slope of -1.2 and $M^{*}\left(b_{\mathrm{J}}\right)=-19.5$, a cluster shape characterized by a King model with a core radius of $0.5 h_{50}^{-1} \mathrm{Mpc}$, and a K-correction of $\Delta b_{\mathrm{J}}=3 z$ (see e.g. Efstathiou et al. 1988; Dalton et al. 1997). The dashed curves in Fig. 18 give then the number of galaxy counts expected for the various magnitude limits. We note that the distribution of the data points are well described by the theoretical curves with a steep rise at low redshift which is due to an increasing part of the cluster being covered by the aperture and the decrease at high redshift when only the very brightest cluster galaxies are detected. We also not the increasing difficulty to recognize clusters above a redshift of $z=0.3$.

Tests based on the comparison of the cluster redshift distribution with simulations involving the REFLEX X-ray luminosity and selection function (in right ascension, declination and redshift) and assuming no evolution of the cluster population with redshift show that there is no significant deficit of clusters in the sample out to a redshift $z=0.3$ (e.g. Fig. 8 in Schuecker et al. 2000). Even beyond a redshift of 0.3 where the detection of clusters in the COSMOS data becomes more difficult, we note no deficit in the cluster population. Calculations based on 


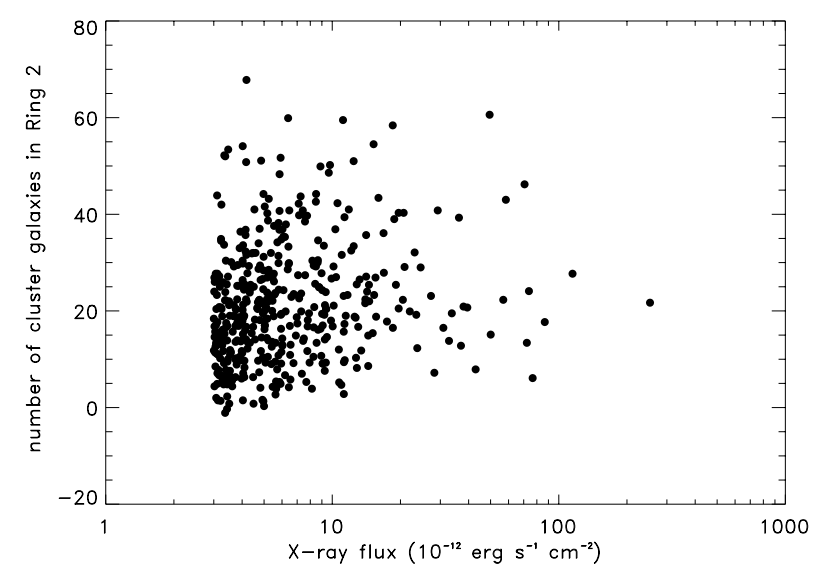

Fig. 17. Distribution of the number of galaxies counted in aperture 2 versus X-ray flux. The background galaxy density has been subtracted from the aperture counts

a no-evolution assumption, in the X-ray luminosity function as derived in the forthcoming paper (Böhringer et al. 2001a), and on the selection function derived here, lead to the prediction of an expectation value for the detection at redshifts beyond $z=0.3$ of 12 clusters, where 11 have been found. This indicates that the most distant clusters in REFLEX are optically rich enough to just be captured by the galaxy count technique applied to the COSMOS data. Even the most distant clusters in the sample, which have independently been found as extended RASS sources, are detected and selected by the correlation based on the COSMOS data. The actual significance value of $1.2 \sigma$ for the extreme case of the most distant REFLEX cluster at $z=0.45$, RXCJ1347.4-1144, (2.5 $\sigma$ for aperture 3$)$ and $0.45 \sigma$ for the second most distant cluster at $z=0.42$ (found in aperture 1 with a $1.4 \sigma$ signal) are quite low for aperture 2, however. Still, the significance in the optimal aperture is surprisingly good for the high redshifts of these clusters.

In summary, we conclude that our combined use of $\mathrm{X}$-ray and optical data leads to a very successful selection of cluster candidates without an introduction of a significant optical bias, and we expect to be over $90 \%$ complete for the chosen X-ray flux limit.

\section{Further classification of the cluster candidates}

After this anticipation of the final results we return to the sequence of the REFLEX sample construction. Up to this point we have compiled a sample of cluster candidates relying only on machine based algorithms (except for the manual exclusion of obvious pathological cases like the bright diffraction spike stellar images and the multiple detections). The only two selection criteria are the X-ray flux limit for the X-ray sources and a signal of a galaxy overdensity in the optical data. This sample has still an estimated contamination of non-cluster X-ray sources of $30-40 \%$ (as discussed in the previous section). For the identification work that follows we treat each source individually, compile as much information as possible, and try

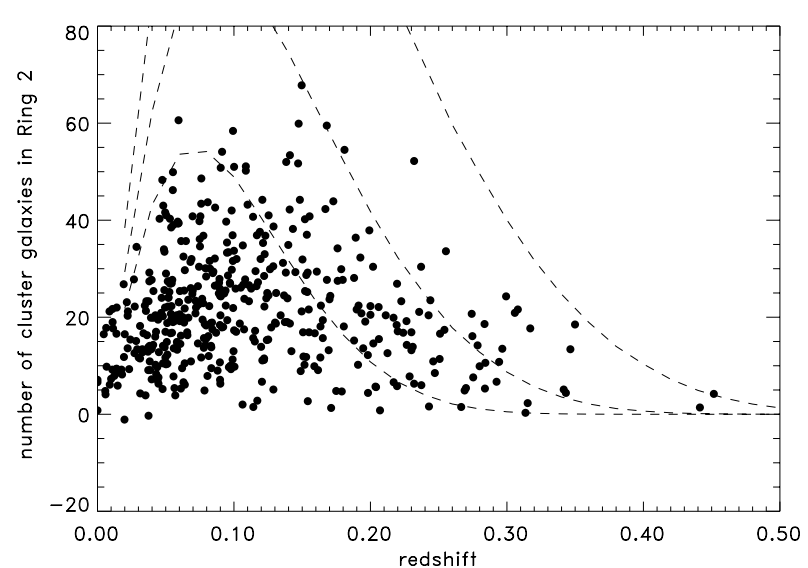

Fig. 18. Distribution of the number of galaxies counted in aperture 2 versus redshift. Also shown are the expected number counts for this aperture for a cluster with an Abell richness of 100 for the optical magnitude limits of $b_{\mathrm{J}}=20,21$, and 22, respectively. For the cluster model used to calculate these expected numbers see the description in the text

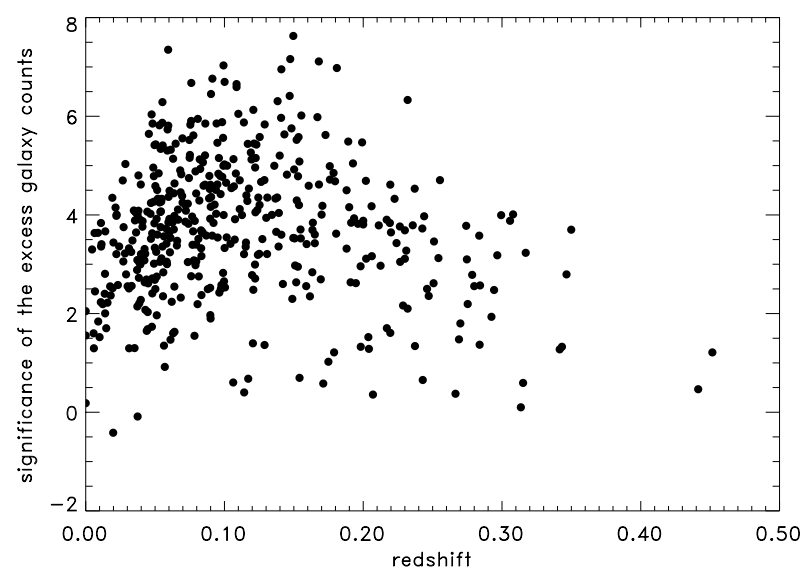

Fig. 19. Distribution of the significance of the galaxy overdensity for the galaxies found in aperture 2 versus redshift. Note that the significance can get negative if the number of galaxies counted in the aperture is less than the background value

to arrive at a safe classification in each case. The types of information used are the X-ray properties of the source, optical images (from the STScI scans of the POSS and UK Schmidt plates or CCD images if available), and literature information (including previous X-ray source identifications).

The basic X-ray source parameters used to assess the source properties are the probability for an extension of the X-ray emission (from the Kolmogorov-Smirnov test mentioned above) and the spectral hardness ratio. The hardness ratio and its photon statistical error is compared to the expected hardness ratio for a thermal cluster spectrum for a temperature of $5 \mathrm{keV}$ and the absorbing interstellar column density at the source position (Dickey \& Lockman 1990), for details see Böhringer et al. (2000). As a measure of the consistency of the observation with $\mathrm{X}$-ray emission from the intracluster plasma of a cluster, 
we take the deviation of the predicted and observed hardness ratio in units of the statistical error (in $\sigma$ units).

In a first step we are discarding all sources that can be unambiguously classified as non-cluster objects. As a safe exclusion criterion we have either accepted a well documented previous identification or combined at least two quality criteria which exclude the identification as a cluster. Thus the following information leads to discarding a cluster candidate source:

i) Positive identification as a non-cluster X-ray source in the literature.

ii) The X-ray source is both well consistent with a point source and deviates by more than $3 \sigma$ from the theoretically predicted hardness ratio of an X-ray cluster. In addition we find no indication of a cluster in the optical images.

iii) The X-ray source is point-like and there is an AGN spectrum observed for a galaxy or a point-like optical object at the X-ray source position.

iv) A point-like X-ray source coincides with a $b_{\mathrm{J}}<$ 12 mag star (within a radius of about 30 arcsec) and there is no cluster visible in the optical image.

A large part of the contamination fraction can be removed from the source list by use of these criteria. The positive identifications are given simply by the observation of a clearly extended X-ray source and a galaxy cluster in the optical images. For all the X-ray sources for which no clear classifications can be obtained and also for all clusters that have not been identified previously and for which no redshift is available, further spectroscopic and if necessary imaging observations were conducted. In total 431 targets were observed by us within the ESO key programme for this project (including candidates with X-ray fluxes below the current flux limit of the REFLEX survey) as well as additional targets in related programmes (e.g. Cruddace et al. 2000 in preparation).

The identification strategy in the optical follow-up observations is similar to the scheme given above. We either try to establish the existence of a galaxy cluster as the counterpart of the X-ray source by securing several coincident galaxy redshifts in the X-ray source field or by arriving at an alternative identification of the X-ray source which is in general an AGN. AGN are found for about $10 \%$ of the sources for which spectra were taken. More details about the identification process and the different types of non-cluster sources found within the ESO key programme will be given in a subsequent paper which will also provide the object catalogue. Here we concentrate on discussing the statistics of some X-ray properties of the cluster and non-cluster X-ray sources in Sect. 10.

\section{Further tests of the sample completeness}

To further test the completeness of the REFLEX sample we have conducted two additional searches for clusters. The first search is based on the X-ray source extent and the second on a systematic search for X-ray emission from Abell clusters.
In the search for clusters among the extended RASS sources we have inspected all sources in the flux limited sample $\left(F_{\mathrm{X}} \geq 310^{-12} \mathrm{erg} \mathrm{s}^{-1} \mathrm{~cm}^{-2}\right)$ which feature a KSprobability less than 0.01 of being point-like and which are not already included in the REFLEX sample. In total 48 additional extended sources are found (after removal of strange detections at exposure edges and fragments of larger clusters already included in REFLEX). 35 of these sources are identified with bright stars and QSO and we notice that they are often borderline cases concerning the extent significance; another fraction of these sources feature an extent in the analysis without deblending because they are close pairs of point sources with identifications other than clusters. The remaining objects for which a cluster identification cannot be ruled out are in total 13 X-ray sources: 8 certain clusters, 2 good looking cluster candidates and 3 fields with no indication for a galaxy cluster and no obvious other identification. We are planning further deeper imaging for the latter sources. Thus we have found about 10 objects in this search which have been missed in the REFLEX compilation. The above result can also be used for another interesting and useful statistic. The 35 partly spuriously extended sources among the non-cluster candidates (plus the 28 extended non-cluster sources mentioned in Sect. 10) if compared to an initial sample of 1050 sources (above the REFLEX cut with extended cluster sources subtracted) implies a failure rate of flagging non-extended X-ray sources erroneously as extended of less than $6 \%$.

The Abell and ACO catalogues (Abell 1958; Abell et al. 1989) contain about 5 times as many objects as the REFLEX sample in the study area. Even so we do not expect a very close match of the two samples, since e.g. the correlation of X-ray luminosity and optical richness is quite weak (see e.g. Ebeling et al. 1993), the large overabundance of Abell clusters provides a good check regarding problems in the recognition of clusters by the galaxy count technique based on COSMOS. To search systematically for X-ray emission from all ACO and ACO supplementary clusters we run the GCA algorithm on all ACO positions allowing for a recentering of the method within a radius of 10 arcmin of the input position. We find only one ACO supplementary cluster that was not flagged by the galaxy counts and should be included in REFLEX given its GCA flux. This cluster was already found in the above discussed additional cluster search at the positions of the extended RASS X-ray sources. It happens that this cluster is actually close to the boundary to the Large Magellanic Cloud which might explain the deficiency in counted galaxies at this position.

Since the search for X-ray emission from ACO clusters is independent of the previous source detection in the RASS II primary source list, we are not only testing the completeness of the cluster finding by the optical galaxy counts but also the source detection in the RASS II standard analysis (Voges et al. 1999). Since we find no ACO cluster missing in REFLEX due to its non-detection in RASS II, we can conclude that missing of sources in 
RASS II is not a significant problem for the completeness of the REFLEX sample. Such completeness of the primary source detection will be studied further by simulations of the source detection efficiency in the RASS data.

In summary, from the available material we find a missing fraction of clusters of about $2-3 \%$ in REFLEX which can be recovered as described in this section. This small fraction is still well consistent with the internal estimate of a completeness of over $90 \%$ and further supports the quality of the REFLEX sample. Note that the additional cluster detections are not integrated into the REFLEX sample to conserve its homogeneity but will be listed as REFLEX supplementary clusters in forthcoming catalogue publications.

\section{Properties of the REFLEX cluster sample}

After the identification based on the spectroscopic and imaging follow-up observations 452 objects were accepted as galaxy clusters in the catalogue. For three objects of this list there is no conclusive redshift available yet and two of these three objects are still classified as candidates which require a final confirmation. Figure 5 summarizes again the steps which led to the construction of this sample and the two side branches used to test the sample completeness in form of a flow diagram.

Figure 20 shows the distribution of the X-ray luminosities and redshifts for the 449 clusters with redshift information. Details on the way the fluxes and luminosities of the clusters are calculated can be obtained from Böhringer et al. (2000, 2001a). The parabolic boundary in the plot reflects the flux limit of the sample. The sample is covering a luminosity range from about $110^{42} \mathrm{erg} \mathrm{s}^{-1}$ to $610^{45} \mathrm{erg} \mathrm{s}^{-1}$. The objects with luminosities below $10^{43} \mathrm{erg} \mathrm{s}^{-1}$ are Hickson type groups and even smaller units down to elliptical galaxies with extended X-ray halos. In the latter objects the extended X-ray emission is still tracing a massive dark matter halo which is in principle not different from a scaled down cluster. Therefore we have included them in the cluster sample with the caveat that we are not certain at present how well the population of these objects below a luminosity of $10^{43} \mathrm{erg} \mathrm{s}^{-1}$ is sampled in this project. This is because some of them feature a very small membership number which may not always guarantee that they are detected by the galaxy count search.

At high redshifts, beyond $z=0.3$, only exceptionally luminous objects are observed, with X-ray luminosities of several $10^{45} \mathrm{erg} \mathrm{s}^{-1}$. Even in this simple distribution plot we can recognize inhomogeneities in the cluster distribution which can be attributed in a more detailed analysis to the large-scale structure of the Universe (Collins et al. 2000; Schuecker et al. 2000). The paucity of the data at very low redshifts in Fig. 20 is an effect of the small sampling volume. The apparent deficiency of clusters with $L_{x} \geq 10^{45} \mathrm{erg} \mathrm{s}^{-1}$ in the redshift interval $z=0-0.15$ is certainly an effect of large-scale structure. Only about 3 such X-ray luminous clusters are expected in this region.

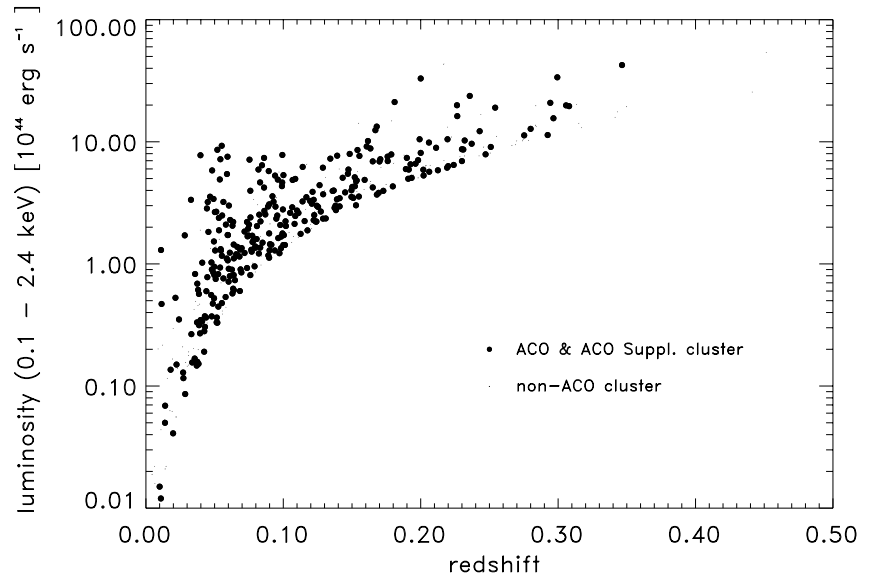

Fig. 20. Distribution of the REFLEX sample clusters in redshift and X-ray luminosity. The clusters catalogued by Abell et al. (1989) and the non-ACO clusters are marked differently. The luminosities are calculated for a Hubble constant of $50 \mathrm{~km} \mathrm{~s}^{-1} \mathrm{Mpc}^{-1}$

While we do not expect the sample to be complete above a redshift of $z=0.3$, the expected number of objects at these high redshifts is indeed very small in a no-evolution model. We explore this further in a forthcoming paper.

Figure 20 also shows which of the clusters in the luminosity redshift distribution are clusters already catalogued by Abell et al. (1989) and which are mostly new. Since the difference of the two different populations is not so easily recognized in this figure we have plotted the nonAbell clusters separately in Fig. 21. One notes that the non-ACO clusters are distributed over the whole range of parameters covered by the total REFLEX sample. As we had expected, many non-ACO clusters are found among the nearby low luminosity, poor clusters which fail Abell's richness threshold and among the most distant clusters, which are not covered well in the optical plates. To our surprise there is also a large fraction of non-ACO clusters found in the intermediate redshift range with X-ray luminosities implying more typical Abell type cluster masses. These latter clusters indicate an incompleteness effect in the Abell catalogue. A similar result was found for the northern BCS sample as shown in Ebeling et al. (1998).

Since we do not have a homogeneous exposure coverage of the REFLEX survey area as described in Sect. 3 we have to apply a corresponding correction to any statistical study of the REFLEX sample. The best way to take the effect of the varying exposure and the effect of the interstellar absorption into account is to calculate for each sky position the number of photons needed to reach a certain flux limit. This includes both the exposure and the sensitivity modification by interstellar extinction. In total the sensitivity variation due to extinction is less than a factor of 1.25 in the REFLEX survey area (see also Böhringer et al. 2000 for details and numerical values). The so defined sensitivity distribution across the REFLEX study region is shown in Fig. 22. Since for the relatively short exposures in the RASS the source detection process is 


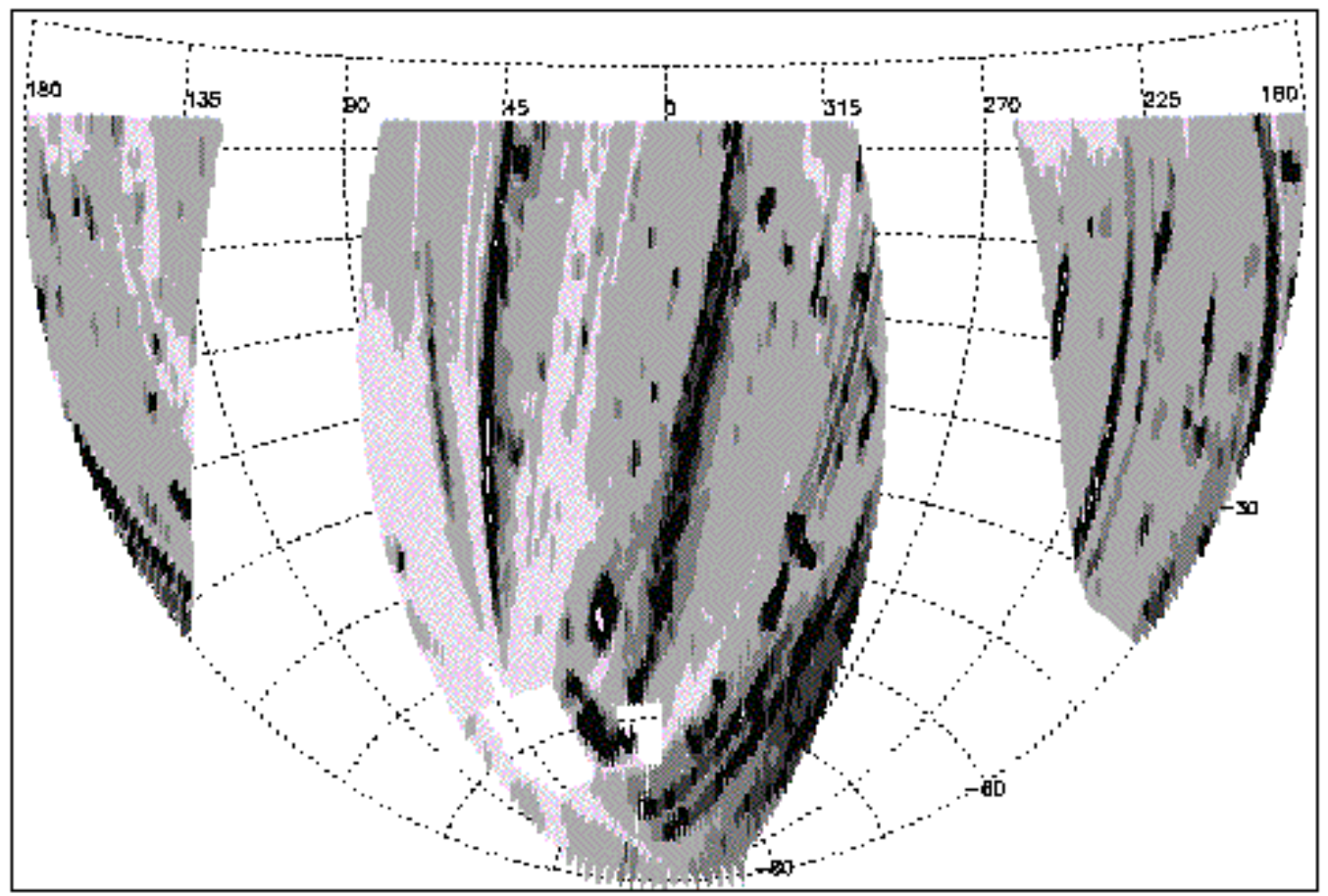

Fig. 22. Sensitivity map of RASS II in the area of the REFLEX survey. Five levels of increasing grey scale have been used for the coding the sensitivity levels given in units of the number of photons detected at the flux limit: $>60,30-60,20-30,15-20$, and $<15$, respectively. The coordinate system is equatorial for the epoch J2000

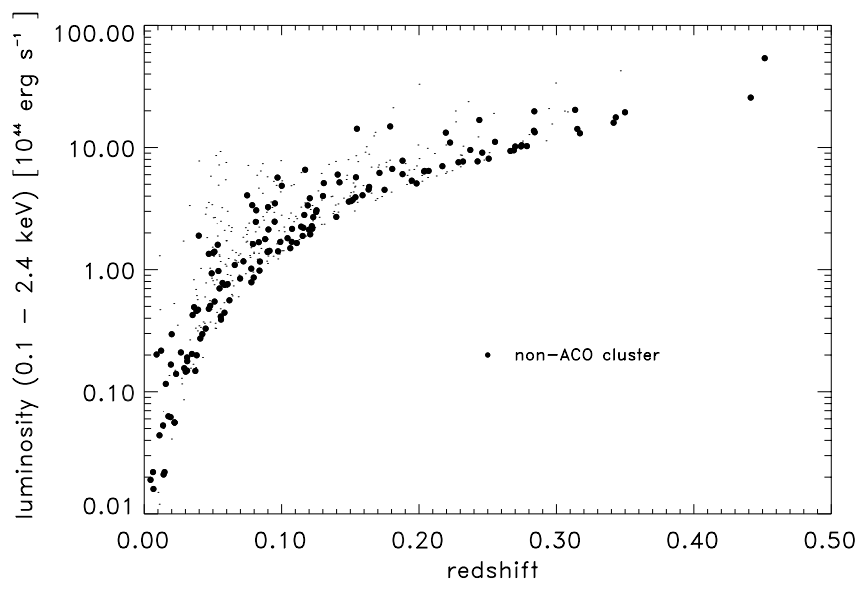

Fig. 21. Distribution of the non-ACO clusters in the REFLEX sample in redshift and X-ray luminosity. These clusters cover practically the whole distribution range of all REFLEX clusters. The clusters catalogued by Abell et al. (1989) are also shown as very light points

practically always source photon limited and not background limited (except for the most diffuse, low-surface brightness structures) the success rate of detection depends mostly on the number of photons. The use of the ROSAT hard band to characterize the cluster emission further reduces the background which is a great advantage for this analysis. Thus fixing a minimum number of photons per source we can calculate the effective survey depth in terms of the flux limit at any position on the sky. The integral of this survey depth versus sky coverage is shown in Fig. 23 for the three cases of a minimum detec- tion of 10,20 , and 30 photons. Also shown is the nominal flux limit of $310^{-12} \mathrm{erg} \mathrm{s}^{-1} \mathrm{~cm}^{-2}$. We note that for a detection requirement of 10 photons the sky coverage is $97 \%$ at a flux limit of $310^{-12} \mathrm{erg} \mathrm{s}^{-1} \mathrm{~cm}^{-2}$. For the much more conservative requirement of at least 30 photons per source the sky coverage for the nominal flux limit of the survey is about $78 \%$. For the remaining part of the survey area the flux limit is slightly reduced. Since the sensitivity map is available for the whole study area (Fig. 22) we can for any choice of the minimum number of photons calculate the correction for the missing sky coverage as a function of flux also for the three-dimensional analyses e.g. the determination of the correlation function and the power spectrum of the cluster density distribution (see Collins et al. 2000; Schuecker et al. 2000).

In Fig. 24 we give the integral surface number counts of clusters for the REFLEX sample as a function of $\mathrm{X}$-ray flux ( $\log N-\log S$-curve). For this determination we have chosen the conservative requirement of a minimum of 30 counts. The figure also shows the result of a maximum likelihood fit of a power law function to the data for the corrected fluxes. The likelihood analysis takes the uncertainties of the flux measurement (analogous to the description of Murdoch et al. 1973) and the variations of the effective sky coverage for a count limit of 30 photons (as given in Fig. 23) into account. The resulting power law index is constraint to the range -1.39 with a $1 \sigma$ error of \pm 0.07 . The normalization in Fig. 24 is fixed to be consistent with the total number of clusters found. This result is in good agreement within the errors with other determinations of the cluster number counts as the results by 


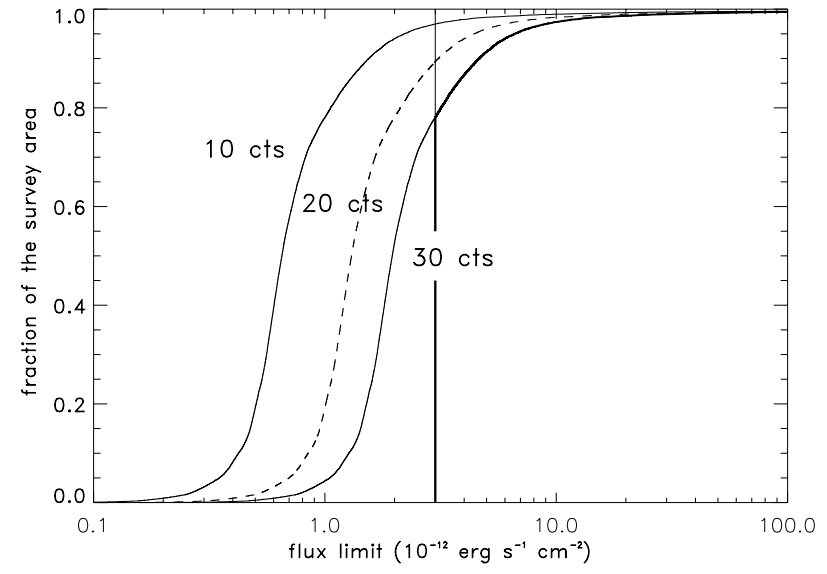

Fig. 23. Effective sky coverage of the REFLEX sample. The thick line gives the effective sky area for the nominal flux limit of $310^{-12} \mathrm{erg} \mathrm{s}^{-1} \mathrm{~cm}^{-2}$ and a minimum number of 30 photons per source as used e.g. for the correction of the $\log N-\log S$ curve shown in Fig. 24. For further details see text

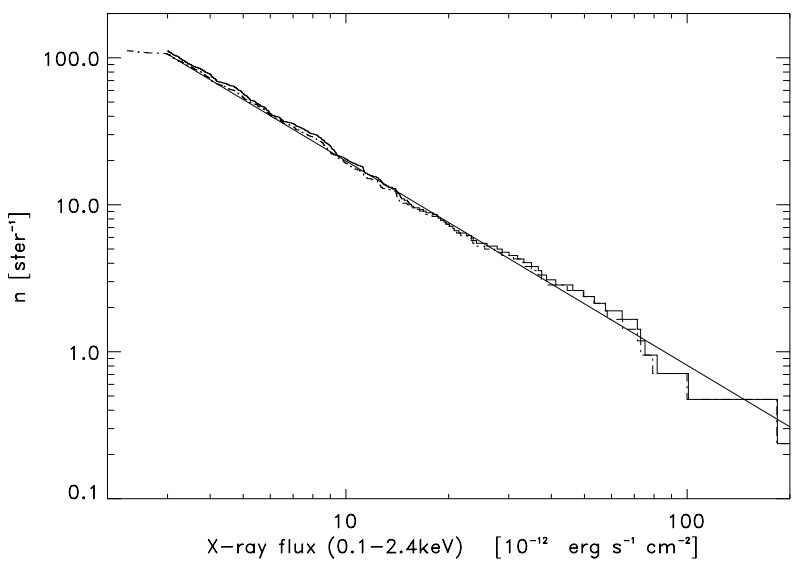

Fig. 24. $\log N$-Log $S$-distribution of the REFLEX sample clusters. The solid line shows the $\log N-\log S$ function for the nominal fluxes (determined for an assumed temperature of $5 \mathrm{keV}$ and $z=0$ ) which is used for the REFLEX flux cut while the dashed line shows the same function for the corrected fluxes as described in Sect. 2. The straight line shows the result of a maximum likelihood fit of a power law function to the data yielding a slope value of $-1.39( \pm 0.07)$

Ebeling et al. (1998); De Grandi et al. (1999) and Rosati et al. (1998). Note that the flux values used correspond to the observed fluxes. The currently best estimate for the total flux implies an average correction by a factor of about 1.1. The fact that the observed $\log N-\log S$-distribution follows the straight line so closely down to the lowest fluxes shows clearly that there is no significant incompleteness effect close to the flux limit.

Given the $\log N-\log S$-distribution corrected for the varying flux limit as shown in Fig. 24, we can now also calculate the number of clusters we expect to be detected with a certain number of counts. This distribution is shown in Fig. 25. Here we are first of all interested in checking the completeness of the sample concerning detections at low photon numbers ( $<30$ photons). Since the

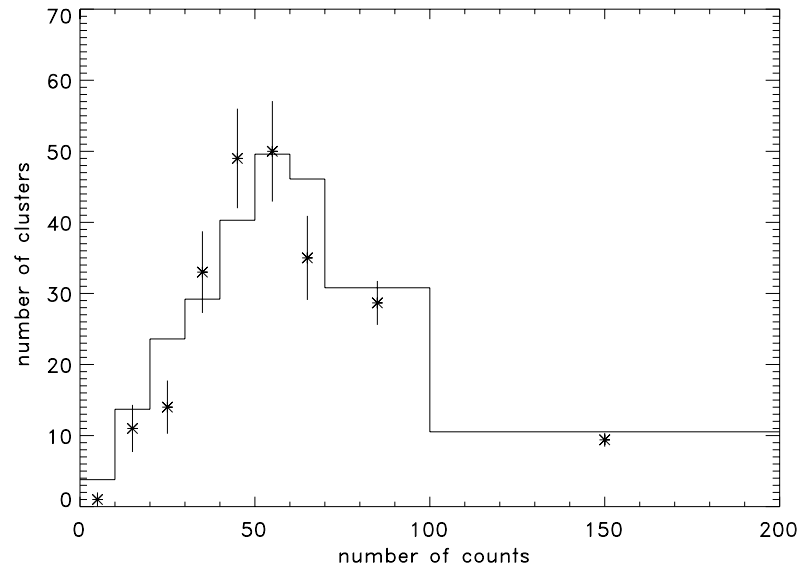

Fig. 25. Distribution of the number of source counts per cluster for the REFLEX sample. The numbers are given as the number of objects per bin of ten photons width. The solid line gives the expected numbers as calculated from the $\log N-\log S$ distribution while the stars give the actual number counts with their Poissonian errors

$\log N-\log S$-distribution was constructed based on clusters with more than 30 counts only, it provides an independent check on the relative completeness of the sample for low compared to high photon numbers. We note that the number of clusters to be detected with low photon numbers is quite small and also that there is no striking deficit of clusters at low counts. Below a detection with 10 counts 3.8 clusters are expected and 1 is detected. In the interval between a detection of 10 to 20 counts there is no deficit and for the interval between 10 and 30 counts the expectation is about 37 clusters compared to 26 found, a $2 \sigma$ deviation. Therefore we expect very little difference for the statistical analyses using different cuts in count rate, as long as the corresponding sky coverage is taken into account. In fact in the construction of the luminosity function we find only a difference of less than 2 percent (in the fitting parameters for an analysis using a 10 photon count and a 30 photon count limit, respectively (Böhringer et al. 2001a). The proper corrections for the effective sky area will become increasingly important, however, when the sample is extended to lower flux limits.

\section{Statistics of the $X$-ray properties of the cluster sources and the sample contamination}

The GCA X-ray source analysis returns two source quality parameters, the spectral hardness ratio and the source extent. These two parameters are not used for the selection of the candidate sample. We have only used this information in conjunction with optical data as a justification to remove a number of obviously contaminating sources. Therefore the distribution of these source properties gives a practically independent information on the nature of the REFLEX cluster sample and it is interesting to study them in comparison to the properties of the non-cluster sources. (The results for the spectral hardness ratios are used here in comparison to the expected values that can be 
well calculated for cluster type spectra $(5 \mathrm{keV})$ and given interstellar HI column density in the light of sight. The parameter quoted is the difference between the measurement and the prediction in units of $\sigma$ of the measurement uncertainty). In Fig. 27 we show the distribution of the two X-ray parameters for the 452 REFLEX clusters. The figure also shows the boundaries used for the decision as dotted lines: a source is considered to be very likely extended if it has a Kolmogorov-Smirnov probability of less than $0.01(-\log P=2)$; and a deviation from the expected hardness ratio of more than $3 \sigma$ (to the soft side) is considered as an argument against a cluster identification. Based on these cuts we find that $81 \%$ of the REFLEX clusters feature an X-ray source extent. (This sample fraction of course depends on the threshold value used in the KS-test. For a less stringent threshold value of 0.05 for the probability of a source to be point-like we could characterize more than $90 \%$ of the sources as extended. The merits of relaxing the threshold condition are currently tested with Monto Carlo simulations and will be described in a following paper.) How the non-extended sources and their fraction as compared to the REFLEX total are distributed in redshift is shown in Fig. 26. While among nearby objects only for a very small number of groups no significant extent was found, the extent fraction is increasing clearly with redshift. At high redshifts, where only the most luminous clusters are found, still more than half of the clusters feature a measurable extent. Only $6 \%$ of all the sources have an observed spectral parameter which appears too soft (Fig. 27). This is a small failure rate which is partly due to statistical fluctuations, possibily due to an inaccurate acount of the interstellar absorption for some of the sources, and also partly due to the contamination of an AGN in the cluster for some of these few sources. But since the overall deviation is only significant for $6 \%$ of the sources the contamination by AGN which might be indicated here is not a problem for the statistical use of the overall sample.

It is interesting to compare these source parameters with those for non-cluster sources. In Fig. 28 we show the distribution of the hardness ratio deviations and the source extent probabilities for the sample of 221 cluster candidates flagged by the galaxy counts but excluded from the sample in the subsequent identification process. (Note that this sample has some bias in comparison to a random non-cluster sample since e.g. (i) in some cases the optical selection may be due an extended object falsely split up into galaxies (ii) contaminating sources may be preferentially recognized if they have a soft spectrum). There is a large fraction of much softer sources. About 13\% percent feature an apparent extent, however (after a few spurious sources located at exposure edges were removed). This is more than the failure rate typically found in the analysis of a test sample of already identified AGN which are known point sources and the statistics of the falsely flagged extended sources shown in Sect. $8(<6 \%)$. The higher rate of detection of extended sources among these non-cluster sources as compared to the false classification rate found

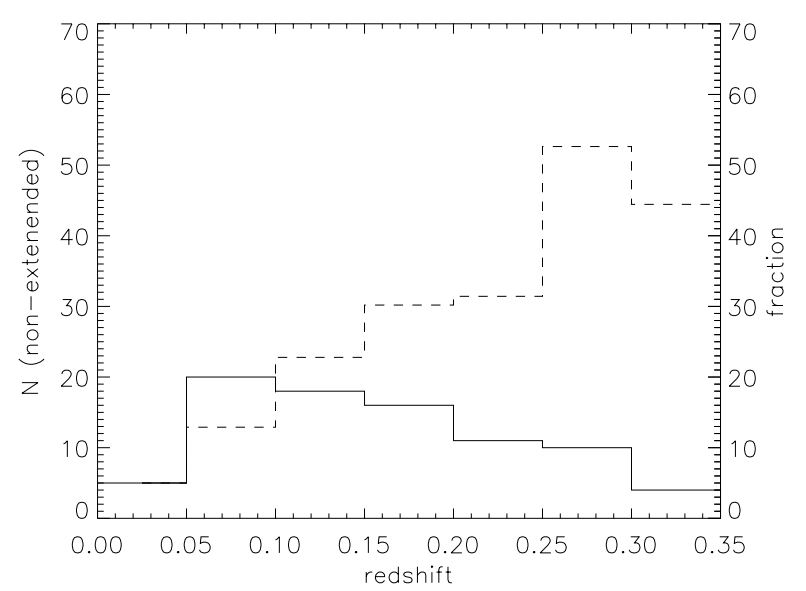

Fig. 26. Redshift distribution of the non-extended X-ray cluster sources in the REFLEX sample (solid line). The fraction of non-extended sources compared to the total REFLEX sample for each redshift bin is shown as dashed line (in units of percent)

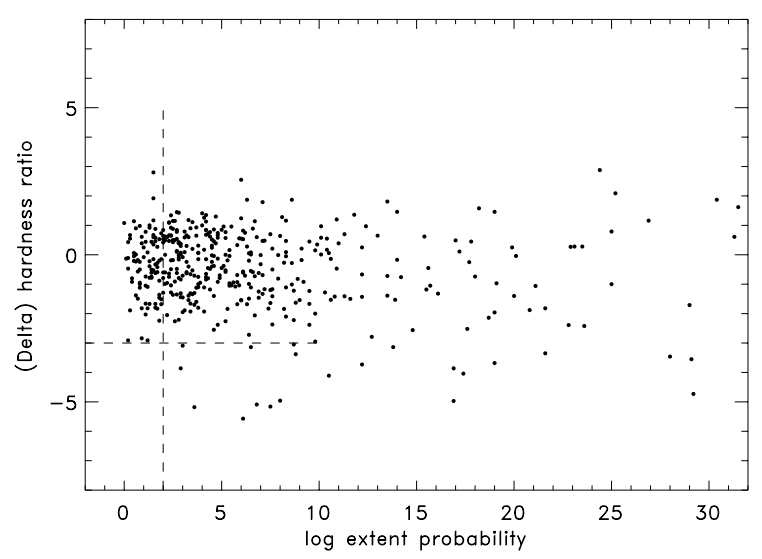

Fig. 27. Hardness ratio deviation and extent probability distribution of the REFLEX clusters. The vertical axis gives the deviation of the measured hardness ratio from the theoretically calculated value in units of the standard deviation. For the detailed definition of the parameters and the threshold values see the text

in Sect. 8 is partly due to really extended X-ray emission from nearby galaxies and due to close, blended double sources. The latter two source types are easily recognized by inspection and therefore the actual false classification rate of point sources as extended including the inspection is at most about half of these $13 \%$.

We can use the difference in the spectral hardness ratio distribution of the two samples of cluster and noncluster sources to test for the possible contamination of the REFLEX cluster sample by AGN which are producing the dominant X-ray emission in a cluster. First of all the high fraction of extended sources guarantees that the emission of most of these $81 \%$ of the X-ray sources is extended emission from the intracluster medium of a cluster. The question is more critical for the non-extended sources. One way of checking the AGN contamination among them is to make a statistical comparison between the spectral 


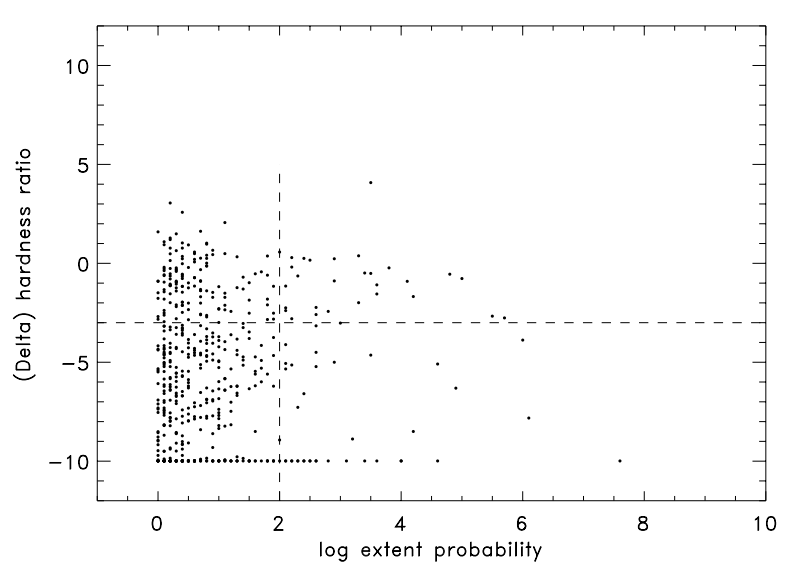

Fig. 28. Hardness ratio deviation and extent probability distribution of stars and AGN excluded from the REFLEX sample clusters

parameters of the extended and non-extended cluster sources in REFLEX. This is done in the form of histograms for the deviation of the measured and expected hardness ratio for the two REFLEX subsamples in Fig. 29. We note that the two distributions are very similar, quite in contrast to the very different distribution of the noncluster sources shown in the same figure. Thus there is no indication that the point-like REFLEX clusters are spectrally significantly different from the extended ones.

This can be more critically tested with cumulative and normalized plots of these same distributions as shown in Fig. 30. Here we note again the similarity of the distributions for the two REFLEX subsamples. They have the same median and the only difference is a slightly broader distribution for the extended cluster sources. We noted this behaviour already for the NORAS cluster sample (Böhringer 2000) and it is most probably due to the fact that the extended sources contain many more photons on average and therefore systematic deviations play an increasing role compared to the pure photon statistics which is the only aspect included in the error calculation. The non-cluster sources labeled $c$ have a completely different distribution. To test the sensitivity of this comparison we have artificially contaminated the point-like X-ray source cluster sample by 20 randomly selected noncluster sources. The resulting distribution function is labeled with an asterisk in Fig. 30. This sample is significantly different from the cluster distribution and such a deviation would easily be recognized. Thus the contamination in the total sample as introduced by the false identification of non-extended REFLEX sources is less than $4 \%$. To this we have to add the possible contamination in the sample of extended sources which could in principle be due to non-cluster sources falsely flagged as extended. Making the following very extreme assumptions: (i) there are as many non-cluster sources as cluster sources in the candidate sample, (ii) the false classification rate is as high as $6 \%$ as found in Sect. 8, (iii) all these falsely classified objects have escaped our careful inspection in the sample cleaning process, we find an upper limit for the possible

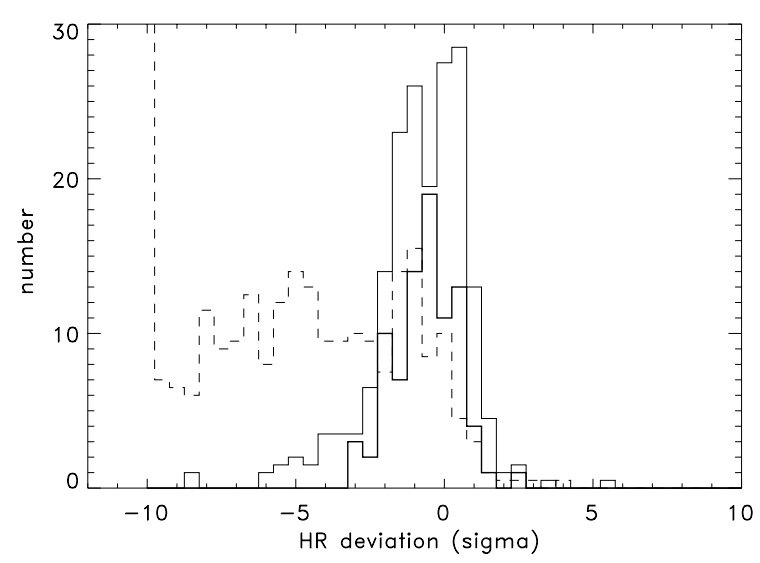

Fig. 29. Distribution of the significance of the deviation of the measured from the expected hardness ratio: extended REFLEX clusters (thin line), REFLEX clusters with no significant extent (thick line), non-cluster sources excluded from the REFLEX sample (dashed line). The plot shows that the extended and non-extended REFLEX clusters do belong to the same spectral class of X-ray sources. Note that the amplitudes of the extended cluster source and non-cluster source histograms have been scale down by a factor of 2 for better comparison with the smaller cluster point source sample

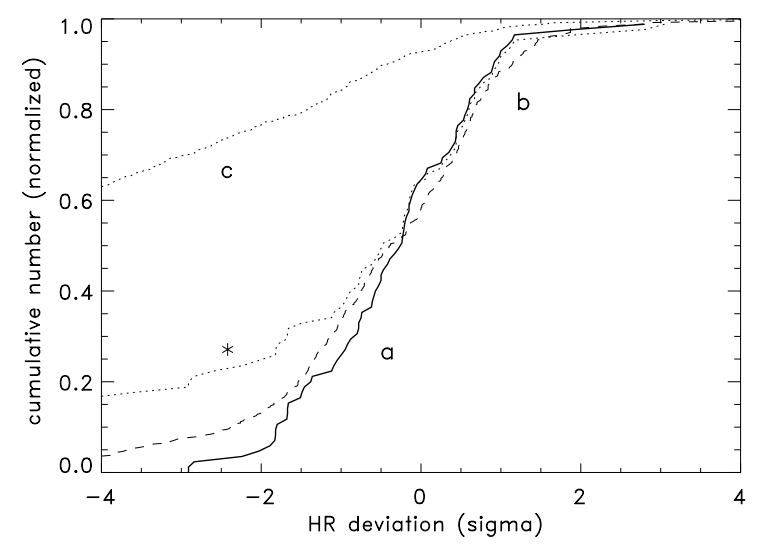

Fig. 30. Cumulative distribution of the significance of the deviation of the measured from the expected hardness ratio: extended REFLEX clusters (dashed line, b), REFLEX clusters with no significant extent (thick line, a), non-cluster sources excluded from the REFLEX sample (c), non-extended REFLEX clusters artificially contaminated by 20 non-cluster sources $\left(^{*}\right)$

contamination of this part of the sample of less than $5 \%$. Therefore the overall contamination cannot be larger than $9 \%$ and is probably much less.

\section{Summary and conclusions}

The main aim of the construction of this X-ray flux-limited galaxy cluster sample is its application to measure the large-scale structure of the Universe and to obtain constraints on cosmological models. To this end the sample has to be very homogenous in all its selection parameters in particular in its coverage of the sky. The unavoidable inhomogeneities have to be well quantified and modeled. Here, we described the construction of the cluster sample 
and the selection function (shown in Fig. 22) and have given the first demonstration that we have achieved our initial goal.

The primary candidate sample has been constructed from the refined second analysis of RASS II and we have used a starting list of detections that includes an overabundance of sources almost down to the $2 \sigma$ detection limit. To ensure that we do not introduce a bias against the flux measurement of extended sources we have reanalysed the sample with the GCA method which accounts for this difficulty (see Böhringer et al. 2000 for checks of this method with deeper X-ray observations). Independent checks for X-ray cluster sources which might have been missed by the source detection in the standard analysis of the RASS by means of the Abell catalogues have not shown a single case of a failed detection.

The second selection is based on a correlation with the galaxy distribution in the COSMOS data base. Alternatively we could have used a combined means of identification of the X-ray sources by correlating also with other galaxy or cluster catalogues to enhance the findings of clusters. The selection based on only one criterion was deliberately chosen because it is the best means to guarantee a fairly homogeneous sampling and to have some control on possible selection effects which can be tested (e.g. we did not find a signature in the correlation of the cluster density with the quality of the plate material to be shown in a following paper in this series). We have actually found an additional small fraction $(\sim 2 \%)$ of clusters which would fulfill the X-ray criteria of the sample as described in Sect. 8, but they are not part of the REFLEX sample to preserve the homogeneity of the present cluster catalogue. The second important point in the optical selection is the achievement of a high completeness. The smaller the missing fraction, the smaller is the imprint of the optical selection criteria on the overall sample. With an estimated completeness in excess of $90 \%$ the imprint should be negligible resulting in an effectively X-ray selected sample of galaxy clusters. This is another important feature of the catalogue since in the following application we will build on the close correlation between X-ray luminosity and cluster mass. The high estimated completeness of the catalogue is to a large part the result of a substantial oversampling of cluster candidates in the correlation process as described in Sects. 5 and 6. (At present we like to limit the statement about the high completeness of the sample to the luminosity range $L_{\mathrm{X}} \geq 10^{43} \mathrm{erg} \mathrm{s}^{-1}$ and redshifts $z \leq 0.3$ until these regimes are explored with further studies.) The price to be paid for this was the large contamination fraction by non-cluster sources of about $30-40 \%$, which required a comprehensive follow-up observation programme.

The following identification work, necessary to remove this substantial contamination has to be very rigorous not to introduce an uncontrolled bias at this step. Therefore the strategy was adopted that either a clear identification could be achieved or in the case of a classification by selection in parameter space at least two strong selec- tion parameters (failure rate not larger than $10 \%$ for each) were required to rule out a cluster identification.

All these measures taken together are the base of the quality of the present sample and its high completeness. The improvement that has been achieved over previous samples can for example be illustrated by a comparison with the northern BCS and extended BCS samples (Ebeling et al. 1998, 2000a). At the flux-limit of BCS, the mean sky surface density of REFLEX clusters is 62.3 ster $^{-1}$ compared to 48.5 ster $^{-1}$ for $\operatorname{BCS}$ ( $78 \%$ of the REFLEX value). At the REFLEX flux-limit the two parameters are 101.3 ster $^{-1}$ (REFLEX) and 70.5 ster $^{-1}$ (extented BCS, $69.5 \%$ of the REFLEX value).

There is still the question of sample contamination. It is for example difficult to rule out in each case that the cluster contains an AGN which is producing the majority of the measured X-ray flux. For this case the standard optical identification, to secure several coincident galaxy redshifts to prove the presence of a cluster, does not help to resolve the situation. The high fraction of true source extents that could be established by our reanalysis and the further tests based on the statistics of the spectral parameter distribution (Sect. 10) show that this is not a serious problem compromising the statistical use of the sample.

We conclude that we have reached the aim of the project to establish a cluster catalogue which can be used for a variety of cosmological studies. Part of these are described in a series of papers already submitted or in preparation covering further tests and the construction of the correlation function (Collins et al. 2000), the power spectrum of the cluster density distribution (Schuecker et al. 2000) the clustering on very large scales (Guzzo et al. 2000), and the X-ray luminosity function (Böhringer et al. 2001a).

Acknowledgements. We thank Joachim Trümper and the ROSAT team providing the RASS data fields and the EXSAS software, Rudolf Dümmler, Harald Ebeling, Andrew Fabian, Herbert Gursky, Silvano Molendi, Marguerite Pierre, Thomas Reiprich, Giampaolo Vettolani, Waltraut Seitter, and Gianni Zamorani for their help in the optical follow-up observations at ESO and for their work in the early phase of the project, and Kathy Romer for providing some unpublished redshifts. We also thank Daryl Yentis and the team at NRL for providing some of the software used in connection with the analysis of the COSMOS data. We have in particular benefited from the use of the COSMOS digitized optical survey of the southern sky, operated by the Royal Observatory Edinburgh. This work has made use of the SIMBAD database operated at CDS, Strasbourg, and of the NASA/IPAC Extragalactic Database (NED) which is operated by the Jet Propulsion Laboratory, California Institute of Technology under contract with NASA. P. S. acknowledges the support by the Verbundforschung under the grant No. 50 OR 970835, H. B. the Verbundforschung under the grand No. 50 OR 93065. L. G. acknowledges financial support by A.S.I. We thank the anonymous referee for helpful comments on the presentation. 


\section{References}

Abadi, M. G., Lambas, D. G., \& Muriel, H. 1998, ApJ, 507, 526

Abell, G. O. 1958, ApJS, 3, 211

Abell, G. O., Corwin, H. G., \& Olowin, R. P. 1989, ApJS, 70, 1

Allen, S. W., Edge, A. C., Fabian, A. C., et al. 1992, MNRAS, 259, 67

Anders, E., \& Grevesse, N. 1989, Geochim. Cosmochim. Acta, 53, 197

Bahcall, N. A. 1988, ARA\&A, 26, 631

Bahcall, N. A., \& Cen, R. 1992, ApJ, 407, L49

Bahcall, N., \& Soneira, 1983, ApJ,

Böhringer, H., \& Wiedenmann, G. 1992, in New Insights into the Universe, ed. V. Martinez, M. Portilla, \& D. Saez, International University Menendez Pelayo Summer School, Valencia, September 1991 (Springer, Heidelberg), 127

Böhringer, H. 1994, in Studying the Universe with Clusters of Galaxies, Proc. of a Workshop at Schloß Ringberg, October 1993, ed. H. Böhringer \& S. Schindler, 93

Böhringer, H., Guzzo, L., Collins, C.A., et al. 1998, The Messenger, No. 94, 21

Böhringer, H., Voges, W., Huchra, J. P., et al. 2000, ApJS, 129, 435 [astroph/0003219]

Böhringer, H., Collins, C. A., Schuecker, P., et al. 2001a, ApJ, to be submitted

Böhringer, H., Schuecker, P., Komossa, S., et al. 2001b, in Mapping the Hidden Universe, Proc. of a workshop in Guanajuato, Mexico, February 2000, ed. R. C. KraanKorteweg, P. A. Henning, \& H. Andernach, in press [astro-ph/0011461]

Borgani, S., Plionis, M., \& Kolokotronis, V. 1999, MNRAS, 305,866

Borgani, S., Rosati, P., Tozzi, P., \& Noman, C. 1998, ApJ, 517, 40

Bryan, G. L., \& Norman, M. L. 1998, ApJ, 495, 80

Burg, R., McLean, B. J., Huchra, J. P., et al. 1994, in Studying the Universe with Clusters of Galaxies, Proc. of a Workshop at Schloß Ringberg, October 1993, ed. H. Böhringer, \& S. Schindler, 133

Burns, J. O., Ledlow, M. J., Loken, C., et al. 1996, ApJ, 467, L49

Cavaliere, A., \& Fusco-Femiano, R. 1976, A\&A, 49, 137

Cen, R., \& Ostriker, J. P. 1994, ApJ, 429, 13

Collins, C. A., Guzzo, L., Nichol, R. C., \& Lumsden, S. L. 1995, MNRAS, 274, 1071

Collins, C. A., Guzzo, L., Böhringer, H., et al. 2000, MNRAS, 319, 939 (Paper II)

Crawford, C. S., Edge, A. C., Fabian, A. C., et al. 1995, MNRAS, 274, 75

Crawford, C. S., Allen, S. W., Ebeling, H., Edge, A. C., \& Fabian, A. C. 1999, MNRAS, 306, 857

Cruddace, R., Hasinger, G., Trümper, J., Schmitt, J. H. M. M., \& Hartner, G. 1991, Experimental Astronomy, 1, 365

Cruddace, R., Voges, W., Böhringer, H., et al. 2000, ApJ, submitted

Dalton, G. B., Efstathiou, G., Maddox, S. J., \& Sutherland, W. J. 1994, MNRAS, 269, 151

Dalton, G. B., Maddox, S. J., Sutherland, W. J., \& Efstathiou, G. 1997, MNRAS, 289, 263

De Grandi, S., Molendi, S., Böhringer, H., \& Voges, W. 1997, ApJ, 486, 738
De Grandi, S., Böhringer, H., Guzzo, L., et al. 1999, ApJ, 514, 148

Dekel, A., Blumenthal, G. R., Primack, J. R., \& Olivier, S. 1989, ApJ, 338, L5

Dickey, J. M., \& Lockman, F. J. 1990, ARAA, 28, 215

Ebeling, H., Voges, W., Böhringer, H., \& Edge, A. C. 1993, A\&A, 275, 360

Ebeling, H., Voges, W., Böhringer, H., et al. 1996, MNRAS, 281,799

Ebeling, H., Edge, A. C., Böhringer, H., et al. 1998, MNRAS, 301,881

Ebeling, H., Edge, A. C., Allen, S. W., et al. 2000a, MNRAS, 318,333

Ebeling, H., Edge, A. C., \& Henry, J. P. 2000b, ApJ, in press [astro-ph/0009101]

Edge, A. C., Stewart, G. C., Fabian, A. C., \& Arnaud, K. A. 1990, MNRAS, 245, 559

Efstathiou, G., Ellis, R. S., \& Peterson, B. A. 1988, MNRAS, 232,431

Einasto, J., Einasto, M., Gottlöber, S., et al. 1997, Nature, 385, 139

Eke, V., Cole, S., \& Frenk, C. S. 1996, MNRAS, 282, 263

Frenk, C. S., White, S. D. M., Efstathiou, G., \& Davis, M. 1990, ApJ, 351, 10

Frenk, C. S., White, S. D. M., Bode, P., et al. 1999, ApJ, 525, 554

Gioia, I. M., Maccacaro, T., Morris, S. L., et al. 1990, ApJS, 72,567

Guzzo, L., Böhringer, H., Briel, U. G., et al. 1995, in Wide-Field Spectroscopy and the Distant Universe, ed. S. J. Maddox, \& A. Aragón-Salamanca (World Scientific, Singapore), 205

Guzzo, L., Böhringer, H., Schuecker, P., et al. 1999, The Messenger, No. 95, 27

Henry, J. P., \& Arnaud, K. A. 1991, ApJ, 372, 410

Henry, J. P., Gioia, I. M., Maccacaro, T., et al. 1992, ApJ, 386, 408

Henry, J. P., Gioia, I. M., Huchra, J. P., et al. 1995, ApJ, 449, 422

Heydon-Dumbleton, N. H., Collins, C. A., \& MacGillivray, H. T. 1989, MNRAS, 238, 379

Kaiser, N. 1986, MNRAS, 222, 323

Klypin, A. A., \& Kopilev, A. I. 1983, Sov. Astron. Lett., 9, 41

Kofman, L., Klypin, A., Pogosyan, D., \& Henry, J. P. 1996, ApJ, 470, 102

Kowalski, M., Cruddace, R. G., Wood, K. S., \& Ulmer, M. P. 1984, ApJS, 56, 403

Lahav, O., Edge, A. C., Fabian, A. C., \& Putney, A. 1989, MNRAS, 238, 88

Lumbsden, S. L., Nichol, R. C., Collins, C. A., \& Guzzo, L. 1992, MNRAS, 258, 1

Lucey, J. R. 1983, MNRAS, 204, 33

Maddox, S. J., Sutherland, W. J., Efstathiou, G., \& Loveday, J. 1990, MNRAS, 243, 692

Markevitch, M. 1998, ApJ, 504, 27

MacGillivray, H. T., \& Stobie, R. S. 1984, Vistas Astr., 27, 433

MacGillivray, H. T., Law, S. D., Cruddace, R. G., et al. 1994, in Cosmological Aspects of X-ray Clusters of Galaxies, ed. W. C. Seitter (Kluwer Academic Publ., Netherlands), 339

Miller, C. J., \& Batuski, D. J. 2000, [astro-ph/0002295]

Moscardini, L., Matarrese, S., De Grandi, S., \& Lucchin, F. 2000, MNRAS, 314, 647

Murdoch, H. S., Crawford, D. F., \& Jauncey, D. L. 1973, ApJ, 183,1 
Nichol, R. C., Briel, U. G., \& Henry, J. P. 1994, MNRAS, 267, 771

Nichol, R. C., Holden, B. P., Romer, A. K., et al. 1997, ApJ, 481,644

Olsen, L. F., Scodeggio, M., Da Costa, L., et al. 1999, A\&A, 345,681

Oukbir, J., \& Blanchard, A. 1992, A\&A, 262, L21

Oukbir, J., \& Blanchard, A. 1997, A\&A, 317, 1

Peacock, J.A. \& West, M. 1992, MNRAS, 259, 494

Piccinotti, G., Mushotzky, R. F., Boldt, E. A., et al. 1982, ApJ, 253,485

Ledlow, M. J., Loken, C., Burns, J. O., Owen, F. N., \& Voges, W. 1999, ApJ, 516, L53

Pierre, M., Böhringer, H., Ebeling, H., et al. 1994, A\&A, 290, 725

Postman, M., Lubin, L. M., Gunn, J. E., et al. 1996, AJ, 111, 615

Press, W. H., \& Schechter, P. 1974, ApJ, 187, 425

Raymond, J. C., \& Smith, B. W. 1977, ApJS, 35, 419

Reiprich, T. H., \& Böhringer, H. 1999, Astron. Nachr., 320, 296

Retzlaff, J., Borgani., S., Gottlöber, S., Klypin, A., \& Müller, V. 1998, New Astron., 3, 631

Romer, A. K., Collins, C. A., Böhringer, H., et al. 1994, Nature, 372,75

Rosati, P., Della Ceca, R., Norman, C., \& Giacconi, R. 1998, ApJ, 492, L21

Sarazin, C. L. 1986, Rev. Mod. Phys., 58, 1

Schuecker, P., Böhringer, H., Guzzo, L., et al. 2000, A\&A (in press, Paper III) [astro-ph/0012105]
Soltan, A., \& Hasinger, G. 1994, A\&A, 288, 77

Stark, A. A., Gammie, C. F., Wilson, R. W., et al. 1992, ApJS, 79,77

Sutherland, W. 1988, MNRAS, 234, 159

Tadros, H., Efstathiou, G., \& Dalton, G. 1998, MNRAS, 296, 995

Thomas, P. A., Colberg, J. M., Couchman, H. M. P., et al. 1998, MNRAS, 296, 1061

Trümper, J. 1992, Royal Astron. Soc. Quart. J., 33, 165

Trümper, J. 1993, Science, 260, 1769

van Haarlem, M. P., Frenk, C. S., \& White, S. D. M. 1997, MNRAS, 287, 817

Viana, P. T. P., \& Liddle 1996, MNRAS, 281, 323

Voges, W., Boller, T., Dennerl, K., et al. 1996, Proc. of the Conference Röntgenstrahlung from the Universe, ed. H. U. Zimmermann, J. E. Trümper, \& H. Yorke, MPE Report No. 263, 637

Voges, W., Aschenbach, B., Boller, T., et al. 1999, A\&A, 349, 389

White, S. D. M., Efstathiou, G., \& Frenk, C. S. 1993, MNRAS, 262,1023

Yentis, D. J., Cruddace, R. G., Gursky, H., et al. 1992, in Digitised Optical Sky Surveys, ed. H. T. MacGillivray, \& E. B. Thomson (Kluwer Academic Publ., Netherlands), 67 Zimmermann, H. U., Becker, W., Belloni, T., et al. 1994, EXSAS User's Guide, MPE Report No. 257

Zwicky, F., Herzog, E., Wild, P., Karpowicz, M., \& Kowal, C. T. 1961-68, Catalogue of galaxies and cluster galaxies, vols. $1-6$ 